\title{
FLUCTUATIONS OF EIGENVALUES OF RANDOM NORMAL MATRICES
}

\author{
YACIN AMEUR, HÅKAN HEDENMALM, and NIKOLAI MAKAROV
}

\begin{abstract}
In this article, we consider a fairly general potential in the plane and the corresponding Boltzmann-Gibbs distribution of eigenvalues of random normal matrices. As the order of the matrices tends to infinity, the eigenvalues condensate on a certain compact subset of the plane-the "droplet." We prove that fluctuations of linear statistics of eigenvalues of random normal matrices converge on compact subsets of the interior of the droplet to a Gaussian field, and we discuss various ramifications of this result.
\end{abstract}

\section{Contents}

1. Notation, preliminaries, and the main result . . . . . . . . . . . 31

2. Further approximations and consequences of Taylor's formula . . . . . . 40

3. The functions $G_{k}$; near-diagonal behavior . . . . . . . . . . . . . . . . 43

4. An expansion formula for the cumulants . . . . . . . . . . . . . . . . . . . . . 48

5. Off-diagonal damping . . . . . . . . . . . . . . . . . . . 52

6. Conclusion of the proof of Theorem $4.4 \ldots \ldots \ldots$. . . . . . . . . . . . . . . . . . . . 58

7. Concluding remarks . . . . . . . . . . . . . . . 65

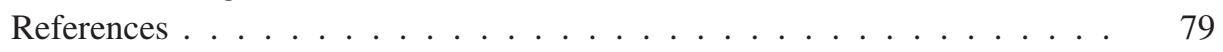

\section{Notation, preliminaries, and the main result}

\section{Random normal matrix ensembles}

Let a weight function (or potential) $Q: \mathbb{C} \rightarrow \mathbb{R}$ be fixed. We assume throughout that $Q$ is $e^{\infty}$ on $\mathbb{C}$ (except sometimes for a finite set, where the value may be $+\infty$ ) and that there are positive numbers $C$ and $\rho$ such that

$$
Q(z) \geq \rho \log |z|^{2}, \quad|z| \geq C .
$$

DUKE MATHEMATICAL JOURNAL

Vol. 159, No. 1, (C) 2011 DOI 10.1215/00127094-1384782

Received 18 October 2009. Revision received 30 October 2010.

2010 Mathematics Subject Classification. Primary 15B52; Secondary 82C22.

Authors' research supported by the Göran Gustafsson Foundation.

Makarov's work partially supported by National Science Foundation grant DMS-0201893. 
Let $\mathfrak{N}_{n}$ be the space of all normal $n \times n$ matrices $M$ (i.e., such that $M^{*} M=$ $M M^{*}$ ) with metric induced from the standard metric on the space $\mathbb{C}^{n^{2}}$ of all $n \times n$ matrices. Write $M=U D U^{*}$, where $U$ is unitary, that is, of class $\mathfrak{U}_{n}$, and where $D=\operatorname{diag}\left(\lambda_{i}\right) \in \mathbb{C}^{n}$.

It is well known (see [11], [14]) that the Riemannian volume form on $\mathfrak{N}_{n}$ is given by $\mathrm{d} M_{n}:=\mathrm{d} U_{n}\left|V_{n}\left(\lambda_{1}, \ldots, \lambda_{n}\right)\right|^{2} \mathrm{~d}^{2} \lambda_{1} \cdots \mathrm{d}^{2} \lambda_{n}$, where $\mathrm{d} U_{n}$ is the normalized $\mathfrak{U}_{n}$-invariant measure on $\mathfrak{U}_{n} / \mathbb{T}$ and where $V_{n}$ is the Vandermonde determinant

$$
V_{n}\left(\lambda_{1}, \ldots, \lambda_{n}\right)=\prod_{j<k}\left(\lambda_{j}-\lambda_{k}\right)
$$

We introduce another parameter $m \geq 1$ and consider the probability measure (on $\left.\mathfrak{N}_{n}\right)$

$$
\mathrm{d} P_{m, n}(M)=\frac{1}{C_{m, n}} e^{-m \text { trace } Q(M)} \mathrm{d} M_{n}
$$

where $C_{m, n}$ is the normalizing constant making the total mass equal to 1 .

In random matrix theory, it is common to study fluctuation properties of the spectrum. In the present case, this means that one disregards the unitary part of $P_{m, n}$ and passes to the following probability measure on $\mathbb{C}^{n}$ (the density of states):

$$
\mathrm{d} \Pi_{m, n}\left(\lambda_{1}, \ldots, \lambda_{n}\right)=\frac{1}{Z_{m, n}}\left|V_{n}\left(\lambda_{1}, \ldots, \lambda_{n}\right)\right|^{2} e^{-m \sum_{j=1}^{n} Q\left(\lambda_{j}\right)} \mathrm{d} A_{n}\left(\lambda_{1}, \ldots, \lambda_{n}\right) .
$$

Here the partition function $Z_{m, n}$ is given by

$$
Z_{m, n}=\int_{\mathbb{C}^{n}}\left|V_{n}\left(\lambda_{1}, \ldots, \lambda_{n}\right)\right|^{2} e^{-m \sum_{j=1}^{n} Q\left(\lambda_{j}\right)} \mathrm{d} A_{n}\left(\lambda_{1}, \ldots, \lambda_{n}\right),
$$

where we put $\mathrm{d} A_{n}\left(\lambda_{1}, \ldots, \lambda_{n}\right)=\mathrm{d} A\left(\lambda_{1}\right) \cdots \mathrm{d} A\left(\lambda_{n}\right) ; \mathrm{d} A(z)=\mathrm{d}^{2} z / \pi$ is the suitably normalized area measure in the plane. (The integral (1.3) converges when $m / n>\rho^{-1}$; we always assume that this is the case.)

Now fix a number $\tau$ such that

$$
0<\tau<\rho .
$$

We can think of the eigenvalues $\left(\lambda_{i}\right)_{1}^{n}$ as a system of point charges (electrons) confined to a plane, under the influence of the external magnetic potential $m Q$ (see [33]). In the limit when $m \rightarrow \infty, n / m \rightarrow \tau$, the growth condition (1.1) on $Q$ is sufficient to force the point charges to condensate on a certain finite portion of the plane, called the droplet, the details of which depend on $Q$ and $\tau$. Thus the system of electrons, the Coulomb gas, lives in the vicinity of the droplet. Inside the droplet the 
repulsive behavior of the point charges takes over and causes them to be very evenly spread out.

\section{The droplet}

We review some elements from weighted potential theory. Let $\Delta$ denote the normalized Laplacian $\Delta=\partial \bar{\partial}$, where $\partial=(1 / 2)\left(\partial_{x}-\mathrm{i} \partial_{y}\right)$ and $\bar{\partial}=(1 / 2)\left(\partial_{x}+\mathrm{i} \partial_{y}\right)$. Write

$$
X=\{\Delta Q>0\}
$$

Let $\mathrm{SH}_{\tau}$ denote the set of subharmonic functions $f: \mathbb{C} \rightarrow \mathbb{R}$ such that $f(z) \leq$ $\tau \log _{+}|z|^{2}+\mathcal{O}(1)$ as $z \rightarrow \infty$. The equilibrium potential $\widehat{Q}_{\tau}$ is defined as the envelope

$$
\widehat{Q}_{\tau}(z)=\sup \left\{f(z) ; f \in \mathrm{SH}_{\tau} \text { and } f \leq Q \text { on } \mathbb{C}\right\} .
$$

The droplet associated with the number $\tau$ is the set

$$
\wp_{\tau}=\left\{Q=\widehat{Q}_{\tau}\right\} .
$$

Our assumptions then imply that $\widehat{Q}_{\tau} \in \mathrm{SH}_{\tau}, \widehat{Q}_{\tau} \in \mathcal{C}^{1,1}(\mathbb{C})$, that $\delta_{\tau}$ is a compact set, and that $\widehat{Q}_{\tau}$ is harmonic outside $\boldsymbol{S}_{\tau}$ (see, e.g., [26] or [18]). In particular, since $z \mapsto \tau \log _{+}\left(|z|^{2} / C\right)-C$ is a subharmonic minorant of $Q$ for large enough $C$, we have

$$
\widehat{Q}_{\tau}(z)=\tau \log _{+}|z|^{2}+\mathcal{O}(1) \quad \text { on } \mathbb{C} \text {. }
$$

Let $\mathcal{P}$ be the convex set of all compactly supported Borel probability measures on $\mathbb{C}$. The energy functional corresponding to $\tau$ is given by

$$
I_{\tau}(\sigma)=\int_{\mathbb{C}^{2}}\left(\log \frac{1}{|z-w|}+\frac{Q(z)+Q(w)}{2 \tau}\right) \mathrm{d} \sigma(z) \mathrm{d} \sigma(w), \quad \sigma \in \mathcal{P} .
$$

Then there exists a unique weighted equilibrium measure $\sigma_{\tau} \in \mathcal{P}$ which minimizes the energy $I_{\tau}(\sigma)$ over all $\sigma \in \mathcal{P}$. Explicitly, this measure is given by

$$
\mathrm{d} \sigma_{\tau}(z)=\tau^{-1} \Delta \widehat{Q}_{\tau}(z) \mathrm{d} A(z)=\tau^{-1} \Delta Q(z) \mathbf{1}_{\tau} \cap X(z) \mathrm{d} A(z) .
$$

(See [26], [18].)

The problem of determining the details of the droplet are known under the names Laplacian growth or quadrature domains. When $Q$ is real analytic in a neighborhood of the droplet, the boundary of the droplet is a finite union of analytic arcs with at most a finite number of singularities which can be either cusps pointing outward from the droplet, or double points, and also possibly a finite set of isolated points (see [19, Section 4], [27]). On the other hand, if $Q$ is just $\bigodot^{\infty}$-smooth, the boundary will in general be quite complicated. 


\section{The correlation kernel}

We state a couple of well-known facts concerning the measure $\Pi_{m, n}$ in (1.2). For positive integers $n$ with $n<m \rho$, we let $H_{m, n}$ be the space of analytic polynomials of degree at most $n-1$ with inner product $\langle f, g\rangle_{m Q}=\int_{\mathbb{C}} f(z) \overline{g(z)} e^{-m Q(z)} \mathrm{d} A(z)$. We denote by $K_{m, n}$ the reproducing kernel for $H_{m, n}$, that is,

$$
K_{m, n}(z, w)=\sum_{j=1}^{n} \phi_{j}(z) \overline{\phi_{j}(w)}
$$

where $\left\{\phi_{j}\right\}_{j=1}^{n}$ is an orthonormal basis for $H_{m, n}$.

It is well known that $\Pi_{m, n}$ is given by a determinant

$$
\begin{aligned}
& \mathrm{d} \Pi_{m, n}\left(\lambda_{1}, \ldots, \lambda_{n}\right)=\frac{1}{n !} \operatorname{det}\left(K_{m, n}\left(\lambda_{i}, \lambda_{j}\right) e^{-m\left(Q\left(\lambda_{i}\right)+Q\left(\lambda_{j}\right)\right) / 2}\right)_{i, j=1}^{n} \\
& \quad \times \mathrm{d} A_{n}\left(\lambda_{1}, \ldots, \lambda_{n}\right) .
\end{aligned}
$$

More generally, for $k \leq n$, the $k$-point marginal distribution $\Pi_{m, n}^{k}$ is the probability measure on $\mathbb{C}^{k}$ which is characterized by

$$
\begin{aligned}
& \int_{\mathbb{C}^{k}} f\left(\lambda_{1}, \ldots, \lambda_{k}\right) \mathrm{d} \Pi_{m, n}^{k}\left(\lambda_{1}, \ldots, \lambda_{k}\right)=\int_{\mathbb{C}^{n}} f\left(\lambda_{\pi(1)}, \ldots, \lambda_{\pi(k)}\right) \\
& \quad \times \mathrm{d} \Pi_{m, n}\left(\lambda_{1}, \ldots, \lambda_{n}\right)
\end{aligned}
$$

whenever $f$ is a continuous bounded function depending only on $k$ variables and whenever $\pi:\{1, \ldots, k\} \rightarrow\{1, \ldots, n\}$ is injective. Evidently, $\Pi_{m, n}=\Pi_{m, n}^{n}$. One then has that

$$
\begin{aligned}
\mathrm{d} & \Pi_{m, n}^{k}\left(\lambda_{1}, \ldots, \lambda_{k}\right)=\frac{(n-k) !}{n !} \operatorname{det}\left(K_{m, n}\left(\lambda_{i}, \lambda_{j}\right) e^{-m\left(Q\left(\lambda_{i}\right)+Q\left(\lambda_{j}\right)\right) / 2}\right)_{i, j=1}^{k} \\
& \times \mathrm{d} A_{k}\left(\lambda_{1}, \ldots, \lambda_{k}\right) .
\end{aligned}
$$

(For proofs of the identities (1.7), (1.9), see, e.g., [23], [18], or the argument in [26, Section IV.7.2].)

The weighted kernel $K_{m, n}(z, w) e^{-m(Q(z)+Q(w)) / 2}$ is known as the correlation kernel or Christoffel-Darboux kernel corresponding to the ensemble.

\section{Linear statistics}

Let us now fix a function $g \in \mathscr{C}_{b}(\mathbb{C})$ and form the random variable ("linear statistic")

$$
\operatorname{trace}_{n} g: \mathbb{C}^{n} \rightarrow \mathbb{C}, \quad\left(\lambda_{j}\right)_{j=1}^{n} \mapsto \sum_{j=1}^{n} g\left(\lambda_{j}\right) .
$$


Let $E_{m, n}$ denote expectation with respect to the measure $\Pi_{m, n}$ on $\mathbb{C}^{n}$. Likewise, if $k \leq n$, we let $E_{m, n}^{k}$ denote expectation with respect to the marginal distribution $\Pi_{m, n}^{k}$. Then by (1.8) and (1.9), we have

$$
\begin{gathered}
E_{m, n}\left(\frac{1}{n} \operatorname{trace}_{n} g\right)=\frac{1}{n} \sum_{j=1}^{n} E_{m, n}^{1}\left(g\left(\lambda_{j}\right)\right)=E_{m, n}^{1}\left(g\left(\lambda_{1}\right)\right) \\
=\frac{1}{n} \int_{\mathbb{C}} g\left(\lambda_{1}\right) K_{m, n}\left(\lambda_{1}, \lambda_{1}\right) e^{-m Q\left(\lambda_{1}\right)} \mathrm{d} A\left(\lambda_{1}\right) .
\end{gathered}
$$

The asymptotics of the right-hand side can be deduced from the following fact (see [18]; see also [5], [14], [15]):

$$
\int_{\mathbb{C}}\left|\frac{1}{n} K_{m, n}(\lambda, \lambda) e^{-m Q(\lambda)}-\tau^{-1} \Delta \widehat{Q}_{\tau}(\lambda)\right| \mathrm{d} A(\lambda) \rightarrow 0 \quad \text { as } m \rightarrow \infty, n / m \rightarrow \tau .
$$

Combining this with (1.10), one obtains the following well-known result.

THEOREM 1.1 ([18, Theorem 1.1])

Let $g \in \mathcal{C}_{b}(\mathbb{C})$. Then

$$
\frac{1}{n} E_{m, n}\left(\operatorname{trace}_{n} g\right) \rightarrow \int_{\mathbb{C}} g(\lambda) \mathrm{d} \sigma_{\tau}(\lambda) \quad \text { as } m \rightarrow \infty, n / m \rightarrow \tau
$$

We now form the random variable (fluctuation about the equilibrium)

$$
\text { fluct }_{n} g=\operatorname{trace}_{n} g-n \int_{\mathbb{C}} g \mathrm{~d} \sigma_{\tau} .
$$

The main problem considered in this paper is determining the asymptotic distribution of fluct $n g$ as $m \rightarrow \infty$ and $n-m \tau \rightarrow 0$ when $g$ is supported in the interior ("bulk") of $\vartheta_{\tau} \cap X$. For this purpose, we use a result due to Berman [5] concerning the near-diagonal bulk asymptotics of the correlation kernel.

\section{Approximating Bergman kernels}

For convenience, we assume that $Q$ be real analytic in the neighborhood of the droplet. This is not a serious restriction (see Remark 1.5 and Section 7.1). (Moreover, the real analytic case is the most interesting one.)

Let $b_{0}(z, w), b_{1}(z, w)$, and $\psi(z, w)$ be the (unique) holomorphic functions defined in a neighborhood in $\mathbb{C}^{2}$ of the set $\left\{(z, \bar{z}) ; z \in \mathcal{S}_{\tau} \cap X\right\}$ such that $b_{0}(z, \bar{z})=\Delta Q(z)$, $b_{1}(z, \bar{z})=(1 / 2) \Delta \log \Delta Q(z)$, and $\psi(z, \bar{z})=Q(z)$ for all $z \in X$. The first-order 
approximating Bergman kernel $K_{m}^{1}(z, w)$ is defined by

$$
K_{m}^{1}(z, w)=\left(m b_{0}(z, \bar{w})+b_{1}(z, \bar{w})\right) e^{m \psi(z, \bar{w})}
$$

for all $z, w$ where it makes sense, namely, in a neighborhood of the antidiagonal $\left\{(z, \bar{z}) ; z \in \delta_{\tau} \cap X\right\}$.

LEMMA 1.2 (see [5])

Let $K$ be a compact subset of $\oiint_{\tau}^{\circ} \cap X$, and fix $z_{0} \in K$. There then exist numbers $m_{0}$, $C$, and $\varepsilon>0$ independent of $z_{0}$ such that, for all $m \geq m_{0}$ and all $n \geq m \tau-1$, the following holds:

$$
\left|K_{m, n}(z, w)-K_{m}^{1}(z, w)\right| e^{-m(Q(z)+Q(w)) / 2} \leq C m^{-1}, \quad z, w \in D\left(z_{0} ; \varepsilon\right) .
$$

In particular,

$$
\left|K_{m, n}(z, z) e^{-m Q(z)}-\left(m \Delta Q(z)+\frac{1}{2} \Delta \log \Delta Q(z)\right)\right| C m^{-1}, \quad z \in K .
$$

A proof of the result in the present form appears in [1, Theorem 2.8], using essentially the method of Berman [5] and the approximate Bergman projections constructed in [7] (compare also [9], [8]). See [5, Section 1.3], for a comparison with the line bundle setting.

We note that corresponding uniform estimates in Lemma 1.2, up to the boundary of the droplet, are false.

Expectation of fluctuations

Using Lemma 1.2, we can easily prove the following result.

THEOREM 1.3

Suppose that $g \in \mathcal{C}_{0}^{\infty}\left(\S_{\tau}^{\circ} \cap X\right)$. Then

$$
E_{m, n} \text { fluct }_{n} g \rightarrow \int_{\mathbb{C}} g \mathrm{~d} \nu \quad \text { as } m \rightarrow \infty \text { and } n-m \tau \rightarrow 0,
$$

where $v$ is the signed measure

$$
\mathrm{d} v(z)=\frac{1}{2} \Delta \log \Delta Q(z) \mathbf{1}_{\delta_{\tau} \cap X}(z) \mathrm{d} A(z)
$$


Proof

By (1.12), we have

$$
\begin{aligned}
E_{m, n}\left(\text { fluct }_{n} g\right)= & n E_{m, n}^{1} g\left(\lambda_{1}\right)-n \int_{\mathbb{C}} g\left(\lambda_{1}\right) \mathrm{d} \sigma_{\tau}\left(\lambda_{1}\right) \\
= & \int_{\text {supp } g}\left(m \Delta Q(z)+\frac{1}{2} \Delta \log \Delta Q(z)+\mathcal{O}\left(m^{-1}\right)\right) \\
& \times g(z) \mathrm{d} A(z)-n \tau^{-1} \int_{\text {supp } g} g(z) \Delta Q(z) \mathrm{d} A(z) \\
= & \left(m-n \tau^{-1}\right) \int g(z) \Delta Q(z) \mathrm{d} A(z) \\
& +\frac{1}{2} \int g(z) \Delta \log \Delta Q(z) \mathrm{d} A(z)+\mathcal{O}\left(m^{-1}\right) .
\end{aligned}
$$

When $m \rightarrow \infty$ and $m-n \tau^{-1} \rightarrow 0$, the expression in the right-hand side converges to $\int_{\mathbb{C}} g \mathrm{~d} \nu$.

\section{Main result}

Let $\nabla=(\partial / \partial x, \partial / \partial y)$ denote the usual gradient on $\mathbb{C}=\mathbb{R}^{2}$. We have the following theorem.

THEOREM 1.4

Let $g \in \mathcal{C}_{0}^{\infty}\left(\wp_{\tau}^{\circ} \cap X\right)$ be a real-valued test function. The random variable fluct $_{n} g$ on the probability space $\left(\mathbb{C}^{n}, \Pi_{m, n}\right)$ converges in distribution when $m \rightarrow \infty$ and $n-m \tau \rightarrow 0$ to a Gaussian variable with expectation $e_{g}$ and variance $v_{g}^{2}$ given by

$$
e_{g}=\int g \mathrm{~d} \nu, \quad v_{g}^{2}=\frac{1}{4} \int|\nabla g|^{2} \mathrm{~d} A .
$$

This theorem is the analog of a result due to Johansson [22], where the Hermitian case is considered. Following Johansson, we note that, in contrast to the situation of the standard central limit theorem, there is no $1 / \sqrt{n}$-normalization of the fluctuations. The variance is thus very small compared to what it would be in the independent and identically distributed case. This means that there must be effective cancelations caused by the repulsive behavior of the eigenvalues. One can interpret Theorem 1.4

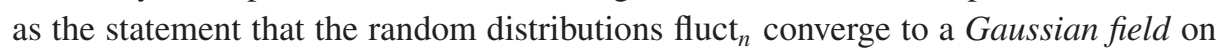
compact subsets of the bulk of the droplet (see Section 7.3).

The formula for $e_{g}$ has already been shown. The rest of this article is devoted to proving the other statements, namely, the formula for $v_{g}^{2}$ and the asymptotic normality of the variables fluct $n g$ when $m \rightarrow \infty$ and $n-m \tau \rightarrow 0$. In the following sections, we assume that $g$ is real-valued. 
We prove Theorem 1.4 using the well-known cumulant method. An alternative approach using an idea of Johansson [22] is sketched in Section 7.2. A comparison between the approaches is found in Remark 7.2.1.

Here we want to mention the parallel work by Berman [6], who independently gave a different proof of a version of Theorem 1.4 valid in a more general situation involving several complex variables.

\section{Remark 1.5}

We emphasize that in our (cumulant-based) proof of Theorem 1.4, we assume $Q$ to be real analytic in a neighborhood of the droplet. The theorem is, however, also true for example for general $Q: \mathbb{C} \rightarrow \mathbb{R} \cup\{+\infty\}$ which satisfy (1.1) and which are finite and $\mathcal{C}^{\infty}$, except in a finite set where the value is $+\infty$. Since these types of potentials are sometimes useful, we indicate after the proof the modifications needed to make it work in this generality (see Section 7.1).

\section{The cumulant method}

For a real-valued random variable $A$, the cumulants $\ell_{k}(A), k \geq 1$, are defined by

$$
\log \mathbf{E}\left(e^{t A}\right)=\sum_{k=1}^{\infty} \frac{t^{k}}{k !} \boldsymbol{C}_{k}(A)
$$

and $A$ is Gaussian if and only if $\ell_{k}(A)=0$ for all $k \geq 3$. Moreover, $\ell_{2}(A)$ is the variance of $A$.

It was observed by Marcinkiewicz that in order to prove asymptotic normality of a sequence of random variables (i.e., convergence in distribution to a normal distribution), it suffices to prove convergence of all moments or, equivalently, convergence of the cumulants. Indeed, convergence of the moments is somewhat stronger than asymptotic normality.

We now fix a real-valued function $g \in \mathcal{C}_{0}^{\infty}\left(\mathcal{S}_{\tau}^{\circ} \cap X\right)$, and we write $\mathcal{C}_{m, n, k}(g)$ for the $k$ th cumulant of trace ${ }_{n} g$ with respect to the measure $\Pi_{m, n}$. Following Rider and Virág [25], we can write the cumulants as integrals involving the cyclic product

$$
\begin{aligned}
& R_{m, n, k}\left(\lambda_{1}, \ldots, \lambda_{k}\right)=K_{m, n}\left(\lambda_{1}, \lambda_{2}\right) K_{m, n}\left(\lambda_{2}, \lambda_{3}\right) \cdots K_{m, n}\left(\lambda_{k}, \lambda_{1}\right) \\
& \quad \times e^{-m\left(Q\left(\lambda_{1}\right)+\cdots+Q\left(\lambda_{k}\right)\right)} .
\end{aligned}
$$

Namely, with

$$
G_{k}\left(\lambda_{1}, \ldots, \lambda_{k}\right)=\sum_{j=1}^{k} \frac{(-1)^{j-1}}{j} \sum_{k_{1}+\cdots+k_{j}=k, k_{1}, \ldots, k_{j} \geq 1} \frac{k !}{k_{1} ! \cdots k_{j} !} \prod_{l=1}^{j} g\left(\lambda_{l}\right)^{k_{l}}
$$


we have (see [25]; see also [12], [30], [31])

$$
\bigodot_{m, n, k}(g)=\int_{\mathbb{C}^{k}} G_{k}\left(\lambda_{1}, \ldots, \lambda_{k}\right) R_{m, n, k}\left(\lambda_{1}, \ldots, \lambda_{k}\right) \mathrm{d} A_{k}\left(\lambda_{1}, \ldots, \lambda_{k}\right)
$$

Note that if $G_{k}\left(\lambda_{1}, \ldots, \lambda_{k}\right) \neq 0$, then $\lambda_{i} \in \operatorname{supp} g$ for some $i$.

The representation (1.16) was used by Rider and Virág [25] in the case of the Ginibre potential $Q=|z|^{2}$ to prove the desired convergence of the cumulants. In [24], the same authors applied the cumulant method to study some determinantal processes in the model Riemann surfaces, and they proved analogs of Theorem 1.4 for a few other special (radial) potentials.

The methods of [25] and [24] depend on the explicit form of the correlation kernel. In the present case, the explicit kernel is too complicated to be of much use. To circumvent this problem, we use the asymptotics in Lemma 1.2 and also some off-diagonal damping results for the correlation kernel (see Section 5).

We want to emphasize that the result of [25] also covers the situation when $g$ is not necessarily supported in the bulk (in the Ginibre case) and that this situation is not treated in Theorem 1.4. (We have more to say about that case in general in Section 7.4 below.)

The cumulant method is well known and has been used earlier by Soshnikov [30] and Costin and Lebowitz [12], for example, to obtain results on asymptotic normality of fluctuations of linear statistics of eigenvalues from some classical compact groups. The method has also been used in the parallel work on linear statistics of zeros of Gaussian analytic functions initiated by Sodin and Tsirelson [29] and generalized by Shiffman and Zelditch [28]. A brief comparison of these results to those of the present paper is given in Section 7.8.

\section{Other related work}

It should also be noted that Theorem 1.4, as well as the more general Theorem 7.4.1 below, follows from the well-known "physical" arguments due to Wiegmann and others (see, e.g., the survey in [33] and the references therein, as well as [16]).

Results related to fluctuations of eigenvalues of Hermitian matrices are found in Johansson [22] and also in [3], [4], and [17]. A lot of work has been done concerning ensembles connected with the classical compact groups (see, e.g., [13], [21], [30], [32], [12]).

\section{Organization and further results}

Sections 2-6 comprise our cumulant-based proof of Theorem 1.4. In our concluding remarks (Section 7), we state and prove further results. We summarize some of them here. In Section 7.2, we sketch an alternative approach to Theorem 1.4 based on a variational argument in the spirit of Johansson [22] (details will appear in [2]). In 
Section 7.4 (Theorem 7.4.1), we state without proof the full plane version of Theorem 1.4 (the proof will appear in [2]). In Section 7.5, we prove universality under the natural scaling: if $m=n$, then for a fixed $z_{0} \in \wp_{1}^{\circ} \cap X$, the rescaled point process $\left(\lambda_{j}\right)_{j=1}^{n} \mapsto\left(\sqrt{n \Delta Q\left(z_{0}\right)}\left(\lambda_{j}-z_{0}\right)\right)_{j=1}^{n}$ converges to the Ginibre $(\infty)$ determinantal point process as $n \rightarrow \infty$. In Section 7.6, we clarify the relation of our present results to the Berezin transform (which we studied in [1]); in particular, we prove the "wavefunction conjecture" (see [18]) that $\left|P_{n}\right|^{2} e^{-n Q} \mathrm{~d} A$ converges to harmonic measure at $\infty$ with respect to $\widehat{\mathbb{C}} \backslash \delta_{1}$, where $P_{n}$ is the $n$th orthonormal polynomial corresponding to the weight $e^{-n Q}$ and where $\widehat{\mathbb{C}}=\mathbb{C} \cup\{\infty\}$.

\section{Further approximations and consequences of Taylor's formula}

In this preparatory section, we discuss a variant of the near-diagonal bulk asymptotics for the correlation kernel and for the functions $R_{n, m, k}$ (see (1.14)), especially for $k=2,3$; such estimates are easily obtained by inserting the asymptotics in Lemma 1.2, and they are used in Section 6.

In this and the following sections, we assume that $Q$ is real analytic near the droplet, except when otherwise specified. Recall that $\psi$ denotes the holomorphic extension of $Q$ from the antidiagonal, that is, $\psi(z, \bar{z})=Q(z)$.

It is well known and easy to show that $\psi$ is determined in a neighborhood of a point at the antidiagonal by the series

$$
\psi(z+h, \overline{z+k})=\sum_{i, j=0}^{\infty} \partial^{i} \bar{\partial}^{j} Q(z) \frac{h^{i} \bar{k}^{j}}{i ! j !}
$$

for $h$ and $k$ in a neighborhood of zero.

For clarity of the exposition, it is worthwhile here to explicitly write down the first few terms in the series for $\psi$ and $Q$

$$
\begin{aligned}
\psi(z+h, \overline{z+k})= & Q(z)+\partial Q(z) h+\bar{\partial} Q(z) \bar{k}+\frac{1}{2}\left(\partial^{2} Q(z) h^{2}+\bar{\partial}^{2} Q(z) \bar{k}^{2}\right) \\
& +\Delta Q(z) h \bar{k}+\text { "higher-order terms" }
\end{aligned}
$$

and

$$
\begin{aligned}
Q(z+h)= & Q(z)+\partial Q(z) h+\bar{\partial} Q(z) \bar{h}+\frac{1}{2}\left(\partial^{2} Q(z) h^{2}+\bar{\partial}^{2} Q(z) \bar{h}^{2}\right) \\
& +\Delta Q(z)|h|^{2}+\mathcal{O}\left(|h|^{3}\right)
\end{aligned}
$$

for small $|h|$. Using that $\overline{\psi(z, w)}=\psi(\bar{w}, \bar{z})$ and that $Q$ is real analytic near the droplet, it is easy to prove uniformity of the $\mathcal{O}$-terms in $z$ when $z \in \mathcal{S}_{\tau}$. This means 
that there is $\varepsilon>0$ such that

$$
\begin{aligned}
& \left.\left.|2 \operatorname{Re} \psi(z+h, \bar{z})-Q(z)-Q(z+h)+\Delta Q(z)| h\right|^{2}|\leq C| h\right|^{3}, \\
& \quad z \in \mathcal{S}_{\tau},|h| \leq \varepsilon
\end{aligned}
$$

In the following we consider $h$ such that $|h| \leq M \delta_{m}$, where $M$ is fixed and where

$$
\delta_{m}=\log m / \sqrt{m} .
$$

We then infer from (2.1) that there is a number $C$ depending only on $M$ such that

$$
\begin{aligned}
& \left.|2 m \operatorname{Re} \psi(z+h, \bar{z})-m Q(z)-m Q(z+h)+m \Delta Q(z)| h\right|^{2} \mid \\
& \quad \leq C m \delta_{m}^{3}, \quad z \in \delta_{\tau},|h| \leq M \delta_{m} .
\end{aligned}
$$

Next, recall the definition of the approximating kernel $K_{m}^{1}(z, w)=$ $\left(m b_{0}(z, \bar{w})+b_{1}(z, \bar{w})\right) e^{m \psi(z, \bar{w})}$ (see Lemma 1.2). We obviously have

$$
\begin{aligned}
& \left|b_{0}(z+h, \bar{z})-\Delta Q(z)\right| \leq C \delta_{m} \quad \text { and } \quad\left|b_{1}(z+h, \bar{z})\right| \leq C \quad \text { when } \\
& \quad z \in \mathcal{S}_{\tau},|h| \leq M \delta_{m},
\end{aligned}
$$

for all large $m$ with $C$ depending only on $K$ and $M$. It follows that

$$
\begin{aligned}
K_{m}^{1}(z+h, z) e^{-m(Q(z+h)+Q(z)) / 2}= & m\left(\Delta Q(z)+\mathcal{O}\left(\delta_{m}\right)\right) e^{m(\psi(z+h, \bar{z})-(Q(z)+Q(z+h)) / 2)}, \\
& z \in \mathcal{S}_{\tau},|h| \leq M \delta_{m},
\end{aligned}
$$

when $m \rightarrow \infty$. Here the $\mathcal{O}$-term is uniform in $z \in \mathcal{S}_{\tau}$. Lemma 1.2 now implies the following estimate for the correlation kernel.

\section{LEMMA 2.1}

Fix a compact subset $K \subset 8_{\tau}^{\circ} \cap X$. Then for all $z \in K$, we have that

$$
\begin{aligned}
& \left|K_{m, n}(z+h, z)\right| e^{-m(Q(z+h)+Q(z)) / 2} \\
& \quad=m\left(\Delta Q(z)+\mathcal{O}\left(\delta_{m}\right)\right) e^{-m \Delta Q(z)|h|^{2} / 2+\mathcal{O}\left(\log ^{3} m / \sqrt{m}\right)}+\mathcal{O}\left(m^{-1}\right), \quad|h| \leq M \delta_{m},
\end{aligned}
$$

when $m \rightarrow \infty$ and $n \geq m \tau-1$; the $\mathcal{O}$-terms are uniform in $z$ for $z \in K$.

We need a consequence concerning the functions $R_{m, n, k}$ for $k=2$ and $k=3$. 


\section{LEMMA 2.2}

Let $K$ be a compact subset of $8_{\tau}^{\circ} \cap X$. Then for $z \in K$, we have

$$
\begin{aligned}
& R_{m, n, 2}(z, z+h)=m^{2}\left(\Delta Q(z)^{2}+\mathcal{O}\left(\delta_{m}\right)\right) e^{-m \Delta Q(z)|h|^{2}+\mathcal{O}\left(\log ^{3} m / \sqrt{m}\right)}+\mathcal{O}(1), \\
& \quad|h| \leq M \delta_{m},
\end{aligned}
$$

and

$$
\begin{aligned}
R_{m, n, 3}\left(z, z+h_{1}, z+h_{2}\right) & \\
= & m^{3}\left(\Delta Q(z)^{3}+\mathcal{O}\left(\delta_{m}\right)\right) e^{m \Delta Q(z)\left(h_{1} \bar{h}_{2}-\left|h_{1}\right|^{2}-\left|h_{2}\right|^{2}\right)+\mathcal{O}\left(\log ^{3} m / \sqrt{m}\right)} \\
& +\mathcal{O}\left(1+m\left(e^{-m \Delta Q(z)\left|h_{1}\right|^{2} / 2}+e^{-m \Delta Q(z)\left|h_{2}\right|^{2} / 2}\right)\right),\left|h_{1}\right|,\left|h_{2}\right| \leq M \delta_{m}
\end{aligned}
$$

when $m \rightarrow \infty$ and $n \geq m \tau-1$; the $\mathcal{O}$-terms are uniform for $z \in K$.

\section{Proof}

The estimate (2.3) follows from Lemma 2.1 since $R_{m, n, 2}(z, z+h)=$ $\left|K_{m, n}(z, z+h)\right|^{2} e^{-m(Q(z)+Q(z+h))}$.

To estimate $R_{m, n, 3}\left(z, z+h_{1}, z+h_{2}\right)$, we first consider the approximation

$$
\begin{aligned}
R_{m, 3}^{1}\left(z, z+h_{1}, z+h_{2}\right)= & K_{m}^{1}\left(z, z+h_{1}\right) K_{m}^{1}\left(z+h_{1}, z+h_{2}\right) K_{m}^{1}\left(z+h_{2}, z\right) \\
& \times e^{-m\left(Q(z)+Q\left(z+h_{1}\right)+Q\left(z+h_{2}\right)\right)}
\end{aligned}
$$

obtained by replacing $K_{m, n}$ by $K_{m}^{1}$ in the definition of $R_{m, n, 3}$.

In view of (2.2), we have, for $z \in K$ and $\left|h_{1}\right|,\left|h_{2}\right| \leq M \delta_{m}$, that

$$
\begin{aligned}
R_{m, 3}^{1}\left(z, z+h_{1}, z+h_{2}\right)= & m^{3}\left(\Delta Q(z)^{3}+\mathcal{O}\left(\delta_{m}\right)\right) \\
& \left.\times e^{m\left(\psi\left(z, \overline{z+h_{1}}\right)\right.}+\psi\left(z+h_{1}, \overline{z+h_{2}}\right)+\psi\left(z+h_{2}, \bar{z}\right)-Q(z)-Q\left(z+h_{1}\right)-Q\left(z+h_{2}\right)\right),
\end{aligned}
$$

where $\mathcal{O}$ is uniform in $z \in K$. A simple calculation with the Taylor expansions for $Q$ at $z$ and $\psi$ at $(z, \bar{z})$ now yields that

$$
\begin{aligned}
\psi\left(z, \overline{z+h_{1}}\right) & +\psi\left(z+h_{1}, \overline{z+h_{2}}\right)+\psi\left(z+h_{2}, \bar{z}\right)-Q(z)-Q\left(z+h_{1}\right) \\
& -Q\left(z+h_{2}\right)=\Delta Q(z)\left(h_{1} \bar{h}_{2}-\left|h_{1}\right|^{2}-\left|h_{2}\right|^{2}\right)+\mathcal{O}\left(|h|_{\infty}^{3}\right)
\end{aligned}
$$

as $h \rightarrow 0$, where we have put $|h|_{\infty}=\max \left\{\left|h_{1}\right|,\left|h_{2}\right|\right\}$. Since the estimate is uniform for $z \in K$, we may use (2.5) to conclude that

$$
\begin{aligned}
& R_{m, 3}^{1}\left(z, z+h_{1}, z+h_{2}\right)=m^{3}\left(\Delta Q(z)^{3}+\mathcal{O}\left(\delta_{m}\right)\right) \\
& \quad \times e^{m \Delta Q(z)\left(h_{1} \bar{h}_{2}-\left|h_{1}\right|^{2}-\left|h_{2}\right|^{2}\right)+\mathcal{O}\left(\log ^{3} m / \sqrt{m}\right)}, \quad|h|_{\infty} \leq M \delta_{m},
\end{aligned}
$$


when $m \rightarrow \infty$, and again the $\mathcal{O}$-terms are uniform for $z \in K$. Combining this with Lemma 2.1 and (2.3), and also using the estimate

$$
\left|K_{m, n}\left(z+h_{1}, z+h_{2}\right)\right| e^{-m\left(Q\left(z+h_{1}\right)+Q\left(z+h_{2}\right)\right) / 2} \leq C m
$$

for $|h|_{\infty} \leq M \delta_{m}, n \geq m \tau-1, m$ large (this follows from Lemma 1.2), we readily obtain (2.4).

\section{The functions $G_{k}$; near-diagonal behavior}

In this section, we let $g$ be any sufficiently smooth (sometimes real-valued) function on $\mathbb{C}$ (i.e., not necessarily supported in $\wp_{\tau}^{\circ} \cap X$ ). We then form the corresponding function $G_{k}$ by (1.15). Here, $k \geq 2$ is fixed.

We now analyze the function $G_{k}$ in a neighborhood of the diagonal

$$
\triangle_{k}=\left\{\lambda \mathbf{1}_{k} \in \mathbb{C}^{k} ; \lambda \in \mathbb{C}\right\},
$$

where

$$
\mathbf{1}_{k}=(1,1, \ldots, 1) \in \mathbb{C}^{k}
$$

Our results in this section state that $G_{k}$ vanishes identically on $\triangle_{k}$ and that $G_{k}$ is harmonic at each point of $\triangle_{k}$. This depends on combinatorial identities of a type which were considered earlier in related contexts, for example, by Soshnikov [30] and Rider and Virág ([25], [24]). The following lemma is equivalent to [30, (1.14), p. 1356].

\section{LEMMA 3.1}

For any function $g: \mathbb{C} \rightarrow \mathbb{C}$ and any $k \geq 2$, it holds that $G_{k}=0$ on $\triangle_{k}$.

\section{Proof}

Evidently, we have

$$
G_{k}\left(\lambda \mathbf{1}_{k}\right)=g(\lambda)^{k} \sum_{j=1}^{k} \frac{(-1)^{j-1}}{j} \sum_{k_{1}+\cdots+k_{j}=k, k_{1}, \ldots, k_{j} \geq 1} \frac{k !}{k_{1} ! \cdots k_{j} !} .
$$

The last sum is the number of partitions of $k$ distinguishable elements into $j$ distinguishable, nonempty subsets. Thus (see, e.g., [10, Theorem 9.1, p. 340)], we have

$$
\sum_{k_{1}+\cdots+k_{j}=k, k_{1}, \ldots, k_{j} \geq 1} \frac{k !}{k_{1} ! \cdots k_{j} !}=j ! S(k, j),
$$


where

$$
S(k, j)=\frac{1}{j !} \sum_{r=0}^{j}(-1)^{r}\left(\begin{array}{l}
j \\
r
\end{array}\right)(j-r)^{k}
$$

is the Stirling number of the second kind. Evidently, $S(k, 0)=0$ for $k \geq 1$. Moreover, the well-known recurrence relation for those Stirling numbers (see, e.g., [10, Theorem $8.9,(8.32)])$, gives

$$
S(k-1,0)=\sum_{r=0}^{k-1}(-1)^{r} r ! S(k, r+1)=\sum_{j=1}^{k} \frac{(-1)^{j-1}}{j} j ! S(k, j) .
$$

The lemma follows, since $S(k-1,0)=0$ when $k \geq 2$.

Note that the lemma is equivalent to the following:

$$
\sum_{j=1}^{k} \frac{(-1)^{j-1}}{j} \sum_{k_{1}+\cdots+k_{j}=k, k_{1}, \ldots, k_{j} \geq 1} \frac{1}{k_{1} ! \cdots k_{j} !}=0, \quad k=2,3, \ldots
$$

We note the following simple but rather useful consequence of Lemma 3.1.

\section{LEMMA 3.2}

Let $g \in \mathcal{C}^{1}(\mathbb{C} \rightarrow \mathbb{C})$, and let $k \geq 2$. Then for all $\lambda \in \mathbb{C}$, the following holds:

$$
\left.\sum_{i=1}^{k}\left(\partial_{i} G_{k}\right)\left(\lambda_{1}, \ldots, \lambda_{k}\right)\right|_{\lambda_{1}=\ldots=\lambda_{k}=\lambda}=\left.\sum_{i=1}^{k}\left(\bar{\partial}_{i} G_{k}\right)\left(\lambda_{1}, \ldots, \lambda_{k}\right)\right|_{\lambda_{1}=\cdots=\lambda_{k}=\lambda}=0 .
$$

Proof

By Lemma 3.1, we have $G_{k}\left(\lambda \mathbf{1}_{k}\right)=0$, whence

$$
0=\frac{\partial}{\partial \lambda} G_{k}\left(\lambda \mathbf{1}_{k}\right)=\sum_{i=1}^{k}\left(\partial_{i} G_{k}\right)\left(\lambda \mathbf{1}_{k}\right)
$$

The statement about $\bar{\partial}$ is analogous.

We now turn to a more nontrivial fact. Let us denote by

$$
\Delta_{k}=\partial_{1} \bar{\partial}_{1}+\cdots+\partial_{k} \bar{\partial}_{k}
$$

the Laplacian on $\mathbb{C}^{k}$. 
In the next lemma, we calculate $\Delta_{k} G_{k}$ at every point of the diagonal $\triangle_{k}$ when $k \geq 2$. When $k \geq 3$, we see that $\Delta_{k} G_{k}$ vanishes on the diagonal, which means that $G_{k}$ is nearly harmonic close to the diagonal.

\section{LEMMA 3.3}

Let $g \in \mathcal{C}^{2}(\mathbb{C} \rightarrow \mathbb{R})$, and let $k \geq 2$. Then for all $\lambda \in \mathbb{C}$, we have

$$
\left.\left(\Delta_{2} G_{2}\right)\left(\lambda_{1}, \lambda_{2}\right)\right|_{\lambda_{1}=\lambda_{2}=\lambda}=|\nabla g(\lambda)|^{2} / 2
$$

and

$$
\left.\left(\Delta_{k} G_{k}\right)\left(\lambda_{1}, \ldots, \lambda_{k}\right)\right|_{\lambda_{1}=\ldots=\lambda_{k}=\lambda}=0, \quad k=3,4, \ldots
$$

\section{Proof}

Fix a number $k \geq 2$. Let $1 \leq j \leq k$, and let $k_{1}, \ldots, k_{j}$ be positive integers such that $k_{1}+\cdots+k_{j}=k$. Since, for $1 \leq r \leq j$,

$$
\begin{aligned}
\frac{\partial^{2}}{\partial \lambda_{r} \partial \lambda_{r}^{-}}\left(\prod_{l=1}^{j} g\left(\lambda_{l}\right)^{k_{l}}\right)= & k_{r}\left(k_{r}-1\right) \times \prod_{l=1, l \neq r}^{j} g\left(\lambda_{l}\right)^{k_{l}} \cdot g\left(\lambda_{r}\right)^{k_{r}-2} \cdot \partial g\left(\lambda_{r}\right) \cdot \bar{\partial} g\left(\lambda_{r}\right) \\
& +k_{r} \times \prod_{l=1, l \neq r}^{j} g\left(\lambda_{l}\right)^{k_{l}} \cdot g\left(\lambda_{r}\right)^{k_{r}-1} \cdot \Delta g\left(\lambda_{r}\right),
\end{aligned}
$$

we get (with $\left.\mathbf{1}_{k}=(1, \ldots, 1) \in \mathbb{C}^{k}\right)$

$$
\begin{aligned}
\left(\Delta_{k} G_{k}\right)\left(\lambda \mathbf{1}_{k}\right)= & \sum_{j=1}^{k} \frac{(-1)^{j-1}}{j} \sum_{k_{1}+\cdots+k_{j}=k, k_{1}, \ldots, k_{j} \geq 1} \frac{k !}{k_{1} ! \cdots k_{j} !} \\
& \times\left(g(\lambda)^{k-2}|\bar{\partial} g(\lambda)|^{2} \sum_{r=1}^{j} k_{r}\left(k_{r}-1\right)+g(\lambda)^{k-1} \Delta g(\lambda) \sum_{r=1}^{j} k_{r}\right) .
\end{aligned}
$$

Since $k_{1}+\cdots+k_{j}=k$, the right-hand side of (3.2) simplifies to

$$
\begin{aligned}
& g(\lambda)^{k-2}|\bar{\partial} g(\lambda)|^{2} \sum_{j=1}^{k} \frac{(-1)^{j-1}}{j} \sum_{k_{1}+\cdots+k_{j}=k, k_{1}, \ldots, k_{j} \geq 1} \frac{k !\left(k_{1}\left(k_{1}-1\right)+\cdots+k_{j}\left(k_{j}-1\right)\right)}{k_{1} ! \cdots k_{j} !} \\
& \quad+g(\lambda)^{k-1} \Delta g(\lambda) \sum_{j=1}^{k} \frac{(-1)^{j-1}}{j} \sum_{k_{1}+\cdots+k_{j}=k, k_{1}, \ldots, k_{j} \geq 1} \frac{k \cdot k !}{k_{1} ! \cdots k_{j} !} .
\end{aligned}
$$


Here the last double sum is zero by (3.1), and (3.3) simplifies to

$$
\begin{aligned}
& g(\lambda)^{k-2}|\bar{\partial} g(\lambda)|^{2} \\
& \quad \times \sum_{j=1}^{k} \frac{(-1)^{j-1}}{j} \sum_{k_{1}+\cdots+k_{j}=k, k_{1}, \ldots, k_{j} \geq 1} \frac{k !\left(k_{1}\left(k_{1}-1\right)+\cdots+k_{j}\left(k_{j}-1\right)\right)}{k_{1} ! \cdots k_{j} !} .
\end{aligned}
$$

In order to finish the proof, we must thus show that $S_{2}=2$ and $S_{k}=0$ for all $k \geq 3$, where $S_{k}$ denotes the sum

$$
S_{k}=\sum_{j=1}^{k} \frac{(-1)^{j-1}}{j} \sum_{k_{1}+\cdots+k_{j}=k, k_{1}, \ldots, k_{j} \geq 1} \frac{k !\left(k_{1}\left(k_{1}-1\right)+\cdots+k_{j}\left(k_{j}-1\right)\right)}{k_{1} ! \cdots k_{j} !} .
$$

The case $k=2$ is trivial, so we assume that $k \geq 3$. To this end, we consider exponential generating functions of the form

$$
H_{j}\left(t ; x_{1}, \ldots, x_{j}\right)=\prod_{l=1}^{j}\left(e^{t x_{l}}-1\right)=\sum_{k_{1}=1}^{\infty} \frac{\left(x_{1} t\right)^{k_{1}}}{k_{1} !} \cdots \sum_{k_{j}=1}^{\infty} \frac{\left(x_{j} t\right)^{k_{j}}}{k_{j} !} .
$$

The relevance of this generating function is seen when we expand the product as a power series in $t$ to get

$$
H_{j}\left(t ; x_{1}, \ldots, x_{j}\right)=\sum_{k=1}^{\infty}\left(\sum_{k_{1}+\cdots+k_{j}=k, k_{1}, \ldots, k_{j} \geq 1} \frac{k ! x_{1}^{k_{1}} \cdots x_{j}^{k_{j}}}{k_{1} ! \cdots k_{j} !}\right) \frac{t^{k}}{k !} .
$$

Considering the $x_{j}$ as real variables and denoting

$$
\Delta_{j}^{\mathbb{R}}=\frac{\partial^{2}}{\partial x_{1}^{2}}+\cdots+\frac{\partial^{2}}{\partial x_{j}^{2}}
$$

the Laplacian on $\mathbb{R}^{j}$, we thus obtain

$$
\begin{aligned}
& \Delta_{j}^{\mathbb{R}} H_{j}(t ; 1, \ldots, 1) \\
& \quad=\sum_{k=1}^{\infty}\left(\sum_{k_{1}+\cdots+k_{j}=k, k_{1}, \ldots, k_{j} \geq 1} \frac{k !\left(k_{1}\left(k_{1}-1\right)+\cdots+k_{j}\left(k_{j}-1\right)\right)}{k_{1} ! \cdots k_{j} !}\right) \frac{t^{k}}{k !} .
\end{aligned}
$$

On the other hand, differentiating the product in (3.6) and evaluating at $x_{1}=\cdots=$ $x_{j}=1$ yields

$$
\Delta_{j}^{\mathbb{R}} H_{j}(t ; 1, \ldots, 1)=j t^{2} e^{t}\left(e^{t}-1\right)^{j-1}
$$


Differentiating (3.7) $k$ times with respect to $t$ and evaluating at $t=0$, we obtain the following result:

$\sum_{k_{1}+\cdots+k_{j}=k, k_{1}, \ldots, k_{j} \geq 1} \frac{k !\left(k_{1}\left(k_{1}-1\right)+\cdots+k_{j}\left(k_{j}-1\right)\right)}{k_{1} ! \cdots k_{j} !}=\left.\frac{\mathrm{d}^{k}}{\mathrm{~d} t^{k}}\left(j t^{2} e^{t}\left(e^{t}-1\right)^{j-1}\right)\right|_{t=0}$.

In view of (3.5), this implies that

$$
S_{k}=\left.\frac{\mathrm{d}^{k}}{\mathrm{~d} t^{k}}\left(\sum_{j=1}^{k}(-1)^{j-1} t^{2} e^{t}\left(e^{t}-1\right)^{j-1}\right)\right|_{t=0}=\left.\frac{\mathrm{d}^{k}}{\mathrm{~d} t^{k}}\left(t^{2}\left(1-\left(1-e^{t}\right)^{k}\right)\right)\right|_{t=0}
$$

But since $1-e^{t}=-\left(t+t^{2} / 2 !+t^{3} / 3 !+\cdots\right)$, we see that the coefficients $a_{l}$ in the expansion

$$
t^{2}\left(1-\left(1-e^{t}\right)^{k}\right)=\sum_{l=0}^{\infty} a_{l} t^{l}
$$

must vanish whenever $l \neq 2$ and $l<k+2$. In particular, if, as we have assumed, $k$ is at least 3, then we have $a_{k}=0$, which by (3.9) implies that $S_{k}=0$. The proof is finished.

In addition to the Laplacian $\left(\Delta_{k} G_{k}\right)\left(\lambda \mathbf{1}_{k}\right)$, we also need to consider functions of the form

$$
Z_{k}(\lambda)=\sum_{i<j}\left(\partial_{i} \bar{\partial}_{j} G_{k}\right)\left(\lambda \mathbf{1}_{k}\right), \quad k \geq 2
$$

The following lemma is now easy to prove.

\section{LEMMA 3.4}

We have that $Z_{2}(\lambda)=-|\bar{\partial} g(\lambda)|^{2}$, while $Z_{k}$ is pure imaginary when $k \geq 3$.

\section{Proof}

Again, the case $k=2$ is trivial because $G_{2}\left(\lambda_{1}, \lambda_{2}\right)=g\left(\lambda_{1}\right)^{2}-g\left(\lambda_{1}\right) g\left(\lambda_{2}\right)$. When $k \geq 3$, we may use Lemmas 3.1 and 3.3 to calculate

$$
0=\Delta_{\lambda}\left\{G_{k}\left(\lambda \mathbf{1}_{k}\right)\right\}=\left(\Delta_{k} G_{k}\right)\left(\lambda \mathbf{1}_{k}\right)+\sum_{i \neq j}\left(\partial_{i} \bar{\partial}_{j} G_{k}\right)\left(\lambda \mathbf{1}_{k}\right)=2 \operatorname{Re} Z_{k}(\lambda),
$$

which shows that $Z_{k}$ is pure imaginary. 


\section{An expansion formula for the cumulants}

In this section, we keep a real-valued function $g \in \mathcal{C}_{0}^{\infty}\left(\wp_{\tau}^{\circ} \cap X\right)$ fixed. We reduce the proof of Theorem 1.4 to the proof of another statement (Theorem 4.4 below), which turns out to be easier to handle and which we prove in Section. 6 after a discussion of some basic estimates for $K_{m, n}$ in Section 5 .

To get started, note that an expression for the cumulant $\boldsymbol{C}_{m, n, k}(g)$ was given above in (1.16). It is important to note that (1.16) and the reproducing property of $K_{m, n}$ show that we may also represent the cumulant $\boldsymbol{C}_{m, n, k}(g)$ as an integral over $\mathbb{C}^{k+1}$

$$
\bigodot_{m, n, k}(g)=\int_{\mathbb{C}^{k+1}} G_{k}\left(\lambda_{1}, \ldots, \lambda_{k}\right) R_{m, n, k+1}\left(\lambda, \lambda_{1}, \ldots, \lambda_{k}\right) \mathrm{d} A_{k+1}\left(\lambda, \lambda_{1}, \ldots, \lambda_{k}\right),
$$

where $G_{k}$ and $R_{m, n, k+1}$ are given by (1.15) and (1.14), respectively. Indeed, this simple trick of introducing an extra parameter $\lambda$ into the integral turns out to be of fundamental importance for our proof.

In Section 3, we were able to give a good description of $G_{k}\left(\lambda_{1}, \ldots, \lambda_{k}\right)$ for points near the diagonal $\lambda_{1}=\cdots=\lambda_{k}=\lambda$. For such points, it is natural to write $h_{i}=\lambda_{i}-\lambda$ (where the $\left|h_{i}\right|$ are small) and to work in the coordinate system $\left(\lambda, h_{1}, \ldots, h_{k}\right)$. Indeed, this coordinate system is advantageous for all our purposes. Note that the volume element is invariant with respect to this change of coordinates

$$
\mathrm{d} A_{k+1}\left(\lambda, \lambda_{1}, \ldots, \lambda_{k}\right)=\mathrm{d} A_{k+1}\left(\lambda, h_{1}, \ldots, h_{k}\right)
$$

and that the reproducing property of $K_{m, n}$ is reflected by the fact that

$$
u(\lambda)=\int_{\mathbb{C}} u(h) K_{m, n}(\lambda, \lambda+h) e^{-m Q(\lambda+h)} \mathrm{d} A(h), \quad u \in H_{m, n} .
$$

Thus, with $h=\left(h_{1}, \ldots, h_{k}\right)$ and $\mathbf{1}_{k}=(1, \ldots, 1)$, we can write $(4.1)$ as

$$
\bigodot_{m, n, k}(g)=\int_{\mathbb{C}^{k+1}} G_{k}\left(\lambda \mathbf{1}_{k}+h\right) R_{m, n, k+1}\left(\lambda, \lambda \mathbf{1}_{k}+h\right) \mathrm{d} A_{k+1}(\lambda, h) .
$$

We now fix $\lambda \in \mathbb{C}$, and we use Taylor's formula applied to the function

$$
\mathbb{C}^{k} \rightarrow \mathbb{R} \quad: \quad h \mapsto G_{k}\left(\lambda \mathbf{1}_{k}+h\right)
$$

Since $G_{k}\left(\lambda \mathbf{1}_{k}\right)=0$ by Lemma 3.1, the Taylor series at $h=0$ can be written

$$
G_{k}\left(\lambda \mathbf{1}_{k}+h\right) \sim \sum_{j=1}^{\infty} T_{j}(\lambda, h)
$$


where, in the multi-index notation, we have

$$
T_{j}(\lambda, h)=\sum_{|\alpha+\beta|=j}\left(\partial^{\alpha} \bar{\partial}^{\beta} G_{k}\right)\left(\lambda \mathbf{1}_{k}\right) \frac{h^{\alpha} \bar{h}^{\beta}}{\alpha ! \beta !} .
$$

Note that if $\lambda \notin \operatorname{supp} g$, then $G_{k}$ vanishes identically in a neighborhood of $\lambda \mathbf{1}_{k}$, and so $T_{j}(\lambda, h)=0$ for all $h \in \mathbb{C}^{k}$. Thus the right-hand side of (4.3) is identically zero when $\lambda \notin \operatorname{supp} g$.

Let us write $|h|_{\infty}=\max \left\{\left|h_{1}\right|, \ldots,\left|h_{k}\right|\right\}$. It turns out to be sufficient to consider Taylor series of degree up to 2 . We thus put

$$
G_{k}\left(\lambda \mathbf{1}_{k}+h\right)=T_{1}(\lambda, h)+T_{2}(\lambda, h)+r(\lambda, h),
$$

where $r(\lambda, h)=\mathcal{O}\left(|h|_{\infty}^{3}\right)$ as $h \rightarrow 0$.

The idea now is to replace $G_{k}\left(\lambda \mathbf{1}_{k}+h\right)$ by the right-hand side of (4.4) in the integral (4.2). To simplify matters, we first have the following lemma.

LEMMA 4.1

For all $k \geq 2$, the following holds:

$$
\int_{\mathbb{C}^{k+1}} T_{1}(\lambda, h) R_{m, n, k+1}\left(\lambda, \lambda \mathbf{1}_{k}+h\right) \mathrm{d} A_{k+1}(\lambda, h)=0 .
$$

Proof

First, note that

$$
T_{1}(\lambda, h)=2 \operatorname{Re} \sum_{i=1}^{k}\left(\partial_{i} G_{k}\right)\left(\lambda \mathbf{1}_{k}\right) h_{i}
$$

Integrating termwise in (4.5) with respect to the measure $R_{m, n, k+1}\left(\lambda, \lambda \mathbf{1}_{k}+\right.$ h) $\mathrm{d} A_{k+1}(\lambda, h)$ and observing that the terms on the right-hand side of (4.5) depend only on two variables, the reproducing property of $K_{m, n}$ shows that, for $i=1, \ldots, k$, we have

$$
\begin{array}{r}
\int_{\mathbb{C}^{k+1}}\left(\partial_{i} G_{k}\right)\left(\lambda \mathbf{1}_{k}\right) R_{m, n, k+1}\left(\lambda, \lambda \mathbf{1}_{k}+h\right) h_{i} \mathrm{~d} A_{k+1}(\lambda, h) \\
=\int_{\mathbb{C}^{2}}\left(\partial_{i} G_{k}\right)\left(\lambda \mathbf{1}_{k}\right) R_{m, n, 2}\left(\lambda, \lambda+h_{1}\right) h_{1} \mathrm{~d} A_{2}\left(\lambda, h_{1}\right),
\end{array}
$$


and so we can replace the integral in (4.5) by an integral over $\mathbb{C}^{2}$ (since $R_{m, n, 2}$ is real-valued) to get

$$
\begin{aligned}
& \int_{\mathbb{C}^{k+1}} T_{1}(\lambda, h) R_{m, n, k+1}\left(\lambda, \lambda \mathbf{1}_{k}+h\right) \mathrm{d} A_{k+1}(\lambda, h) \\
& \quad=2 \operatorname{Re} \int_{\mathbb{C}^{2}}\left(\sum_{i=1}^{k}\left(\partial_{i} G_{k}\right)\left(\lambda \mathbf{1}_{k}\right)\right) R_{m, n, 2}\left(\lambda, \lambda+h_{1}\right) h_{1} \mathrm{~d} A_{2}\left(\lambda, h_{1}\right) .
\end{aligned}
$$

The last integral vanishes by Lemma 3.2.

We have now shown that

$$
\bigodot_{m, n, k}(g)=\int_{\mathbb{C}^{k+1}}\left(T_{2}(\lambda, h)+r(\lambda, h)\right) R_{m, n, k+1}\left(\lambda, \lambda \mathbf{1}_{k}+h\right) \mathrm{d} A_{k+1}(\lambda, h) .
$$

To further simplify this expression, we first look more closely at

$$
T_{2}(\lambda, h)=\sum_{|\alpha+\beta|=2}\left(\partial^{\alpha} \bar{\partial}^{\beta} G_{k}\right)\left(\lambda \mathbf{1}_{k}\right) \frac{h^{\alpha} \bar{h}^{\beta}}{\alpha ! \beta !},
$$

which we write in the form

$$
\begin{aligned}
T_{2}(\lambda, h)= & \frac{1}{2} \sum_{i, j=1}^{k}\left(\partial_{i} \partial_{j} G_{k}\right)\left(\lambda \mathbf{1}_{k}\right) h_{i} h_{j}+\frac{1}{2} \sum_{i, j=1}^{k}\left(\bar{\partial}_{i} \bar{\partial}_{j}\right) G_{k}\left(\lambda \mathbf{1}_{k}\right) \bar{h}_{i} \bar{h}_{j} \\
& +\sum_{i, j=1}^{k}\left(\partial_{i} \bar{\partial}_{j} G_{k}\right)\left(\lambda \mathbf{1}_{k}\right) h_{i} \bar{h}_{j}=\operatorname{Re} \sum_{i=1}^{k}\left(\partial_{i}^{2} G_{k}\right)\left(\lambda \mathbf{1}_{k}\right) h_{i}^{2} \\
& +\operatorname{Re} \sum_{i \neq j}\left(\partial_{i} \partial_{j} G_{k}\right)\left(\lambda \mathbf{1}_{k}\right) h_{i} h_{j}+\sum_{i=1}^{k}\left(\partial_{i} \bar{\partial}_{i} G_{k}\right)\left(\lambda \mathbf{1}_{k}\right)\left|h_{i}\right|^{2} \\
& +2 \operatorname{Re} \sum_{i<j}\left(\partial_{i} \bar{\partial}_{j} G_{k}\right)\left(\lambda \mathbf{1}_{k}\right) h_{i} \bar{h}_{j} .
\end{aligned}
$$


Using the reproducing property of $K_{m, n}$, it yields (note that $R_{m, n, k}$ is not real-valued if $k \geq 3$ )

$$
\begin{aligned}
& \int_{\mathbb{C}^{k+1}} T_{2}(\lambda, h) R_{m, n, k+1}\left(\lambda, \lambda \mathbf{1}_{k}+h\right) \mathrm{d} A_{k+1}(\lambda, h) \\
& =\int_{\mathbb{C}^{3}} \operatorname{Re}\left(\sum_{i \neq j}\left(\partial_{i} \partial_{j} G_{k}\right)\left(\lambda \mathbf{1}_{k}\right) h_{1} h_{2}\right) R_{m, n, 3}\left(\lambda, \lambda+h_{1}, \lambda+h_{2}\right) \mathrm{d} A_{3}\left(\lambda, h_{1}, h_{2}\right) \\
& \quad+\operatorname{Re} \int_{\mathbb{C}^{2}}\left(\sum_{i=1}^{k}\left(\partial_{i}^{2} G_{k}\right)\left(\lambda \mathbf{1}_{k}\right)\right) h_{1}^{2} R_{m, n, 2}\left(\lambda, \lambda+h_{1}\right) \mathrm{d} A_{2}\left(\lambda, h_{1}\right) \\
& +2 \int_{\mathbb{C}^{3}} \operatorname{Re}\left(\sum_{i<j}\left(\partial_{i} \bar{\partial}_{j} G_{k}\right)\left(\lambda \mathbf{1}_{k}\right) h_{1} \bar{h}_{2}\right) R_{m, n, 3}\left(\lambda, \lambda+h_{1}, \lambda+h_{2}\right) \mathrm{d} A_{3}\left(\lambda, h_{1}, h_{2}\right) \\
& \quad+\int_{\mathbb{C}^{2}} \sum_{i=1}^{k}\left(\left(\partial_{i} \bar{\partial}_{i} G_{k}\right)\left(\lambda \mathbf{1}_{k}\right)\right)\left|h_{1}\right|^{2} R_{m, n, 2}\left(\lambda, \lambda+h_{1}\right) \mathrm{d} A_{2}\left(\lambda, h_{1}\right) .
\end{aligned}
$$

Let us now introduce some notation. Recall that

$$
\left(\Delta_{k} G_{k}\right)\left(\lambda \mathbf{1}_{k}\right)=\sum_{i=1}^{k}\left(\partial_{i} \bar{\partial}_{i} G_{k}\right)\left(\lambda \mathbf{1}_{k}\right) \quad \text { and } \quad Z_{k}(\lambda)=\sum_{i<j}\left(\partial_{i} \bar{\partial}_{j} G_{k}\right)\left(\lambda \mathbf{1}_{k}\right), \quad \lambda \in \mathbb{C} .
$$

\section{Definition 4.2}

Let us put

$$
\begin{aligned}
A_{m, n}(k)= & \int_{\mathbb{C}^{3}} \operatorname{Re}\left(\sum_{i \neq j}\left(\partial_{i} \partial_{j} G_{k}\right)\left(\lambda \mathbf{1}_{k}\right) h_{1} h_{2}\right) R_{m, n, 3}\left(\lambda, \lambda+h_{1}, \lambda+h_{2}\right) \\
& \mathrm{d} A_{3}\left(\lambda, h_{1}, h_{2}\right), \\
B_{m, n}(k)= & \operatorname{Re} \int_{\mathbb{C}^{2}}\left(\sum_{i=1}^{k}\left(\partial_{i}^{2} G_{k}\right)\left(\lambda \mathbf{1}_{k}\right)\right) h_{1}^{2} R_{m, n, 2}\left(\lambda, \lambda+h_{1}\right) \mathrm{d} A_{2}\left(\lambda, h_{1}\right), \\
C_{m, n}(k)= & 2 \int_{\mathbb{C}^{3}} \operatorname{Re}\left(Z_{k}(\lambda) h_{1} \bar{h}_{2}\right) R_{m, n, 3}\left(\lambda, \lambda+h_{1}, \lambda+h_{2}\right) \mathrm{d} A_{3}\left(\lambda, h_{1}, h_{2}\right), \\
D_{m, n}(k)= & \int_{\mathbb{C}^{2}}\left(\Delta_{k} G_{k}\right)\left(\lambda \mathbf{1}_{k}\right)\left|h_{1}\right|^{2} R_{m, n, 2}\left(\lambda, \lambda+h_{1}\right) \mathrm{d} A_{2}\left(\lambda, h_{1}\right), \quad \text { and } \\
E_{m, n}(k)= & \int_{\mathbb{C}^{k+1}} r(\lambda, h) R_{m, n, k+1}\left(\lambda, \lambda \mathbf{1}_{k}+h\right) \mathrm{d} A_{k+1}(\lambda, h) .
\end{aligned}
$$

Our preceding efforts in this section are then summed up by the following formula. 


\section{LEMMA 4.3}

For all $m, n, k$ and all $g \in \ell_{0}^{\infty}(\mathbb{C})$, we have

$$
\bigodot_{m, n, k}(g)=A_{m, n}(k)+B_{m, n}(k)+C_{m, n}(k)+D_{m, n}(k)+E_{m, n}(k) .
$$

The rest of this article is devoted to a proof of the following theorem.

\section{THEOREM 4.4}

Suppose that $g \in \mathcal{C}_{0}^{\infty}\left(\wp_{\tau}^{\circ} \cap X\right)$. Then for all $k \geq 2$, the numbers $A_{m, n}(k), B_{m, n}(k)$, and $E_{m, n}(k)$ converge to zero as $m \rightarrow \infty$ and $n-m \tau \rightarrow 0$. Moreover, we have that

$$
\lim _{m \rightarrow \infty, n-m \tau \rightarrow 0} D_{m, n}(k)= \begin{cases}\frac{1}{2} \int_{\mathbb{C}}|\nabla g(\lambda)|^{2} \mathrm{~d} A(\lambda) & \text { if } k=2, \\ 0 & \text { if } k \geq 3,\end{cases}
$$

and

$$
\lim _{m \rightarrow \infty, n-m \tau \rightarrow 0} C_{m, n}(k)= \begin{cases}-\frac{1}{4} \int_{\mathbb{C}}|\nabla g(\lambda)|^{2} \mathrm{~d} A(\lambda) & \text { if } k=2, \\ 0 & \text { if } k \geq 3\end{cases}
$$

It should be noted that Theorem 4.4 implies Theorem 1.4. (Convergence of the cumulants of fluct $n g$ to the cumulants of $N\left(e_{g}, v_{g}^{2}\right)$ is equivalent to convergence of the moments, which implies convergence in distribution.)

In order to verify Theorem 4.4, we first need to look more closely at the behavior of the function $(\lambda, h) \mapsto G_{k}\left(\lambda \mathbf{1}_{k}+h\right) R_{m, n, k+1}\left(\lambda, \lambda \mathbf{1}_{k}+h\right)$ in Section 5 . We see that this function becomes negligible when $h$ is "large" in the sense that $\left|h_{i}\right| \geq M_{k} \log m / \sqrt{m}$ for some $i$, where $M_{k}$ is a sufficiently large number independent of $m$ and $n$ as long as supp $g \subset 8_{\tau}^{\circ} \cap X$ and $|n-m \tau| \leq 1$. This implies that we can approximate the integrals defining the numbers $A_{m, n}(k), \ldots, E_{m, n}(k)$ by integrals over a small neighborhood of the diagonal in $\mathbb{C}^{k+1}$.

\section{Off-diagonal damping}

Fix a number $k \geq 2$. Throughout this section, it is convenient to denote

$$
\lambda_{0}=\lambda_{k+1}=\lambda
$$

so that we can write

$$
R_{m, n, k+1}\left(\lambda, \ldots, \lambda_{k}\right)=\prod_{i=0}^{k} K_{m, n}\left(\lambda_{i}, \lambda_{i+1}\right) e^{-m\left(Q\left(\lambda_{i}\right)+Q\left(\lambda_{i+1}\right)\right) / 2} .
$$

We will frequently and without further mention apply this convention in the rest of this section. We need two lemmas. 
LEMMA 5.1 (see [5])

There is a number $C$ such that, for all $z, w \in \mathbb{C}$ and all $m, n$ with $n \leq m \tau+1$, the following holds:

$$
\left|K_{m, n}(z, w)\right|^{2} e^{-m(Q(z)+Q(w))} \leq C m^{2} e^{-m\left(Q(z)-\widehat{Q}_{\tau}(z)\right)} e^{-m\left(Q(w)-\widehat{Q}_{\tau}(w)\right)} .
$$

\section{Proof}

See [5] or [1, Proposition 3.6].

LEMMA 5.2 (see [1])

Let $K$ be a compact subset of $8_{\tau}^{\circ} \cap X$, and let $d=\operatorname{dist}\left(K ; \mathbb{C} \backslash\left(8_{\tau} \cap X\right)\right)$. Then there exist positive numbers $C$ and $\epsilon$ depending only on $d$ such that, for all $z \in K$, $h \in \mathbb{C}$ and all $m, n \geq 1$ such that $|n-m \tau| \leq 1$, the following holds:

$$
\left|K_{m, n}(z, z+h)\right| e^{-m(Q(z)+Q(z+h)) / 2} \leq C m e^{-\epsilon \sqrt{m} \min \{d,|h|\}} .
$$

\section{Proof}

See [1, Theorem 8.3]; see also [6].

It follows from Lemma 5.1 that

$$
\begin{aligned}
& \left|R_{m, n, k+1}\left(\lambda, \lambda_{1}, \ldots, \lambda_{k}\right)\right| \\
& \quad \leq C m^{k+1} e^{-m\left(Q(\lambda)-\widehat{Q}_{\tau}(\lambda)\right)} e^{-m\left(Q\left(\lambda_{1}\right)-\widehat{Q}_{\tau}\left(\lambda_{1}\right)\right)} \cdots e^{-m\left(Q\left(\lambda_{k}\right)-\widehat{Q}_{\tau}\left(\lambda_{k}\right)\right)},
\end{aligned}
$$

when $n \leq m \tau+1$. By the growth assumption (1.1), using that $\tau<\rho$ and equation (1.6), we conclude that there exist positive numbers $C, C^{\prime}$, and $\delta$ such that

$$
\begin{aligned}
& \left|R_{m, n, k+1}\right| \leq C^{\prime} m^{k+1}\left(\max \left\{|\lambda|^{2}, \ldots,\left|\lambda_{k}\right|^{2}\right\}\right)^{-m \delta}, \\
& \text { when } n \leq m \tau+1 \text { and } \max \left\{|\lambda|^{2}, \ldots,\left|\lambda_{k}\right|^{2}\right\} \geq C
\end{aligned}
$$

Thus if $D_{C}(0)$ denotes the polydisk $\left\{\left(\lambda, \ldots, \lambda_{k}\right) ; \max \left\{|\lambda|^{2}, \ldots,\left|\lambda_{k}\right|^{2}\right\} \leq C\right\}$, then, for any $N \in \mathbb{R}$, we have that

$$
\int_{\mathbb{C}^{k+1} \backslash D_{C}(0)}\left(\left|\lambda^{2}+\cdots+\right| \lambda_{k}||^{2}\right)^{N}\left|R_{m, n, k+1}\left(\lambda, \ldots, \lambda_{k}\right)\right| \mathrm{d} A_{k+1}\left(\lambda, \ldots, \lambda_{k}\right) \rightarrow 0,
$$

as $m \rightarrow \infty, n \leq m \tau+1$, when $C$ is large enough. We now show that more is true. First we have the following lemma. In the proofs, we conform to previous notation and write

$$
\delta_{m}=\log m / \sqrt{m} .
$$


We also put

$$
d=\operatorname{dist}\left(\operatorname{supp} g ; \mathbb{C} \backslash\left(\oiint_{\tau} \cap X\right)\right)
$$

and

$$
K=\left\{z \in \mathbb{C} ; \operatorname{dist}\left(z ; \mathbb{C} \backslash\left(\wp_{\tau} \cap X\right)\right) \geq d / 2\right\} .
$$

We also remind the reader of the convention that $\lambda_{k+1}=\lambda_{0}=\lambda$.

\section{LEMMA 5.3}

There exist positive numbers $M, \alpha$, and $m_{0}$ depending only on $k$ and $d$ such that if $\lambda_{j} \in K$ and $\left|\lambda_{j}-\lambda_{j+1}\right| \geq M \delta_{m}$ for some index $j \in\{0, \ldots, k\}$, then for all $m \geq m_{0}$, we have

$$
\left|R_{m, n, k+1}\left(\lambda_{0}, \lambda_{1}, \ldots, \lambda_{k}\right)\right| \leq C m^{-\alpha}, \quad|n-m \tau| \leq 1
$$

where $C$ depends only on $d$.

\section{Proof}

In view of Lemma 5.2, the hypothesis yields that

$$
\left|K_{m, n}\left(\lambda_{j}, \lambda_{j+1}\right)\right| e^{m\left(Q\left(\lambda_{j}\right)+Q\left(\lambda_{j+1}\right)\right) / 2} \leq C m e^{-\epsilon \sqrt{m} \min \left\{d / 2,\left|\lambda_{j}-\lambda_{j+1}\right|\right\}}, \quad|n-m \tau| \leq 1,
$$

with numbers $C$ and $\epsilon$ depending only on $d$, and $\left|\lambda_{j}-\lambda_{j+1}\right| \geq M \delta_{m}$. Choosing $m_{0}$ large enough that $M \delta_{m} \leq d / 2$ for $m \geq m_{0}$, we have that

$$
\left|K_{m, n}\left(\lambda_{j}, \lambda_{j+1}\right)\right| e^{m\left(Q\left(\lambda_{j}\right)+Q\left(\lambda_{j+1}\right)\right) / 2} \leq C m e^{-\epsilon \sqrt{m} M \delta_{m}}=C m^{1-\epsilon M}, \quad|n-m \tau| \leq 1
$$

when $m \geq m_{0}$. On the other hand, if $n \leq m \tau+1$, then Lemma 5.1 yields that

$$
\left|K_{m, n}\left(\lambda_{l}, \lambda_{l+1}\right)\right| e^{-m\left(Q\left(\lambda_{l}\right)+Q\left(\lambda_{l+1}\right)\right) / 2} \leq C m, \quad l=0, \ldots, k .
$$

Now (5.4) and (5.5) imply that

$$
\left|R_{m, n, k+1}\left(\lambda_{0}, \ldots, \lambda_{k}\right)\right|=\prod_{l=0}^{k}\left|K_{m, n}\left(\lambda_{l}, \lambda_{l+1}\right)\right| e^{-m\left(Q\left(\lambda_{l}\right)+Q\left(\lambda_{l+1}\right)\right) / 2} \leq C m^{k+1-\epsilon M}
$$

when $m \geq m_{0}$ and $|n-m \tau| \leq 1$. It now suffices to choose $M$ large enough that

$$
\epsilon M-k-1>0
$$

and then put $\alpha=\epsilon M-k-1$. 
Henceforth, we let $M$ denote a fixed large number with the properties provided by Lemma 5.3. Let us also put

$$
\begin{gathered}
U_{g}(\lambda)=\operatorname{dist}(\lambda ; \operatorname{supp} g), \quad \lambda \in \mathbb{C}, \\
U_{g}^{*}\left(\lambda_{0}, \ldots, \lambda_{k}\right)=\max \left\{U_{g}\left(\lambda_{i}\right) ; i=0, \ldots, k\right\},
\end{gathered}
$$

and

$$
V_{m, k}=\left\{U_{g}^{*}\left(\lambda_{0}, \ldots, \lambda_{k}\right) \geq M k \delta_{m}\right\}
$$

\section{LEMMA 5.4}

The function

$$
\left(\lambda_{0}, \lambda_{1}, \ldots, \lambda_{k}\right) \mapsto G_{k}\left(\lambda_{1}, \ldots, \lambda_{k}\right) R_{m, n, k+1}\left(\lambda_{0}, \lambda_{1}, \ldots, \lambda_{k}\right)
$$

converges to zero uniformly on the set $V_{m, k}$ as $m \rightarrow \infty$ and $|n-m \tau| \leq 1$.

\section{Proof}

Since $G_{k}$ is bounded, it suffices to prove that $R_{m, n, k+1}$ converges to zero uniformly on the set

$$
V_{m, k}^{\prime}=V_{m, k} \cap \operatorname{supp} G_{k}
$$

Here we regard $G_{k}$ as a function of the variables $\lambda_{0}, \ldots, \lambda_{k}$ which is independent of the parameter $\lambda_{0}$. It is then clear that

$$
\operatorname{supp} G_{k} \subset\left\{\left(\lambda_{0}, \ldots, \lambda_{k}\right) ; \lambda_{0} \in \mathbb{C} \text {, and } \lambda_{i} \in \operatorname{supp} g \text { for some } i=1, \ldots, k\right\} \text {. }
$$

Thus if $\left(\lambda_{0}, \ldots, \lambda_{k}\right) \in V_{m, k}^{\prime}$, then there exists an index $i \in\{1, \ldots, k\}$ such that $\lambda_{i} \in \operatorname{supp} g$. Since the function $R_{m, n, k+1}\left(\lambda_{0}, \ldots, \lambda_{k}\right)$ is invariant under the cyclic permutation $0 \mapsto 1 \mapsto \cdots \mapsto k \mapsto 0$ of the indices, we can assume without loss of generality that $i=1$. Then, since $U_{g}\left(\lambda_{1}\right)=0$ and $U_{g}^{*}\left(\lambda_{1}, \ldots, \lambda_{k+1}\right) \geq M k \delta_{m}$, there must exist an integer $j \in\{1, \ldots, k\}$ such that $\left|\lambda_{l}-\lambda_{l+1}\right|<M \delta_{m}$ for all indices $l$ with $1 \leq l<j$ and $\left|\lambda_{j}-\lambda_{j+1}\right| \geq M \delta_{m}$. It then follows from the triangle inequality that

$$
U_{g}\left(\lambda_{j}\right) \leq\left|\lambda_{j}-\lambda_{1}\right|<M k \delta_{m}
$$

If $m$ is large enough that

$$
M k \delta_{m} \leq d / 2,
$$


then (5.8) implies that $\lambda_{j}$ belongs to the compact set $K$ (see (5.3)) and that $\left|\lambda_{j}-\lambda_{j+1}\right| \geq M \delta_{m}$. Hence, Lemma 5.3 yields that

$$
\left|R_{m, n, k+1}\left(\lambda_{0}, \ldots, \lambda_{k}\right)\right| \leq C m^{-\alpha}
$$

for large $m$ when $|n-m \tau| \leq 1$, where $\alpha>0$. This proves that $R_{m, n, k+1}$ converges uniformly to zero on $V_{m, k}^{\prime}$.

Let us now put

$$
N\left(\lambda_{0}, \ldots, \lambda_{k}\right)=\max _{0 \leq i \leq k}\left\{\left|\lambda_{i}-\lambda_{i+1}\right|\right\} .
$$

Next, we prove that the function $G_{k} R_{m, n, k+1}$ is uniformly small on the set

$$
W_{m, k}:=\left\{\left(\lambda_{0}, \ldots, \lambda_{k}\right) ; U_{g}^{*}\left(\lambda_{0}, \ldots, \lambda_{k}\right) \geq M k \delta_{m} \text { or } N\left(\lambda_{0}, \ldots, \lambda_{k}\right) \geq M \delta_{m}\right\},
$$

where $M=M(k, d)$ is a number provided by Lemma 5.4 .

LEMMA 5.5

The function

$$
\left(\lambda_{0}, \lambda_{1}, \ldots, \lambda_{k}\right) \mapsto G_{k}\left(\lambda_{1}, \ldots, \lambda_{k}\right) R_{m, n, k+1}\left(\lambda_{0}, \lambda_{1}, \ldots, \lambda_{k}\right)
$$

converges to zero uniformly on $W_{m, k}$ as $m \rightarrow \infty$, and $|n-m \tau| \leq 1$.

\section{Proof}

By Lemma 5.4, we know that the function (5.10) converges to zero uniformly on the set $\left\{U_{g}^{*} \geq M k \delta_{m}\right\}$. It thus suffices to show uniform convergence on the set

$$
W_{m, k}^{\prime}=\left\{U_{g}^{*}\left(\lambda_{0}, \ldots, \lambda_{k}\right) \leq M k \delta_{m} \text { and } N\left(\lambda_{0}, \ldots, \lambda_{k}\right) \geq M \delta_{m}\right\} .
$$

Now note that if $m$ is large enough that $M k \delta_{m} \leq d / 2$, we have

$$
W_{m, k}^{\prime} \subset K
$$

with $K$ as in (5.3). Hence, if $\left(\lambda_{0}, \ldots, \lambda_{k}\right) \in W_{m, k}^{\prime}$, then we have $\lambda_{i} \in K$ and $\left|\lambda_{i}-\lambda_{i+1}\right| \geq M \delta_{m}$ for some $i$. It then follows from Lemma 5.3 that $\left|R_{m, n, k+1}\left(\lambda_{0}, \ldots, \lambda_{k}\right)\right| \leq C m^{-\alpha}$ when $|n-m \tau| \leq 1$, where $\alpha>0$. It follows that $R_{m, n, k+1} \rightarrow 0$ uniformly on $W_{m, k}^{\prime}$, and the lemma follows.

It is now advantageous to pass to the coordinate system $(\lambda, h)$ where $\lambda=\lambda_{0}$ and $h_{i}=\lambda_{i}-\lambda$ for $i=1, \ldots, k$. Let us put

$$
|h|_{\infty}=\max \left\{\left|h_{i}\right| ; 1 \leq i \leq k\right\}
$$


and

$$
Y_{m, k}=\left\{(\lambda, h) \in \mathbb{C}^{k+1} ; U_{g}(\lambda) \leq M k \delta_{m},|h|_{\infty} \leq M k \delta_{m}\right\}
$$

As we see, everything interesting goes on in the set $Y_{m, k}$ when $m$ is large and $|n-m \tau| \leq 1$.

\section{LEMMA 5.6}

The function

$$
(\lambda, h) \mapsto G_{k}\left(\lambda \mathbf{1}_{k}+h\right) R_{m, n, k+1}\left(\lambda, \lambda \mathbf{1}_{k}+h\right)
$$

converges to zero uniformly on the complement of $Y_{m, k}$ as $m \rightarrow \infty$ and $|n-m \tau| \leq 1$.

\section{Proof}

In view of Lemma 5.5, it suffices to prove that if $(\lambda, h)$ is in the complement of $Y_{m, k}$, then $\left(\lambda, \lambda_{1}, \ldots, \lambda_{k}\right)$ belongs to $W_{m, k}$, where $\lambda_{i}=\lambda+h_{i}$. But if $(\lambda, h) \notin$ $Y_{m, k}$, then either $U_{g}(\lambda)>M k \delta_{m}$ or $\left|\lambda-\lambda_{i}\right|>M k \delta_{m}$ for some $i=1, \ldots, k$. But the latter inequality can only hold if $\left|\lambda_{j}-\lambda_{j+1}\right|>M \delta_{m}$ for some $j$, whence $N\left(\lambda, \lambda_{1}, \ldots, \lambda_{k}\right) \geq M \delta_{m}$. Thus, in either case, we have $\left(\lambda_{0}, \ldots, \lambda_{k}\right) \in W_{m, k}$, and the lemma follows.

The following lemma and subsequent remark contain what is needed to prove the asymptotic behavior of the cumulants in Section 6.

\section{LEMMA 5.7}

We have that

$$
\left.\int_{\mathbb{C}^{k+1} \backslash Y_{m, k}} \mid G_{k}\left(\lambda \mathbf{1}_{k}+h\right) R_{m, n, k+1}\left(\lambda, \lambda \mathbf{1}_{k}+h\right)\right) \mid \mathrm{d} A_{k+1}(\lambda, h) \rightarrow 0
$$

as $m \rightarrow \infty$ and $|n-m \tau| \leq 1$

Proof

It follows from (5.2) that the integrals

$$
I_{m}=\int_{\mathbb{C}^{k+1} \backslash D_{C}(0)} G_{k}\left(\lambda \mathbf{1}_{k}+h\right) R_{m, n, k+1}\left(\lambda, \lambda \mathbf{1}_{k}+h\right) \mathrm{d} A_{k+1}(\lambda, h)
$$

converge absolutely for large enough $m$ and $C$ if $n \leq m \tau+1$, and $I_{m} \rightarrow 0$ as $m \rightarrow \infty$ and $n \leq m \tau+1$. The statement now follows from Lemma 5.6. 


\section{Remark 5.8}

Suppose that $P(\lambda, h)$ is a measurable function on $\mathbb{C}^{k+1}$ such that (i) $P(\lambda, h) \equiv 0$ when $\lambda \notin \operatorname{supp} g$, and (ii) $|P(\lambda, h)| \leq C\left(1+|h|^{2}\right)^{N}$ for some constants $C$ and $N$. (We write $|h|$ for the $\ell^{2}$ norm on $\mathbb{C}^{k}$ so that $|h|_{\infty}^{2} \leq|h|^{2} \leq k|h|_{\infty}^{2}$.)

As above, we can then conclude that

$$
\begin{aligned}
& \int_{\mathbb{C}^{k+1} \backslash Y_{m, k}} P(\lambda, h) R_{m, n, k+1}\left(\lambda, \lambda \mathbf{1}_{k}+h\right) \mathrm{d} A_{k+1}(\lambda, h) \rightarrow 0 \quad \text { as } m \rightarrow \infty, \\
& \quad|n-m \tau| \leq 1 .
\end{aligned}
$$

Indeed, (5.12) follows from Lemma 5.3 if we also use (6.1) to estimate the part of integral over $|h| \geq C$ for $C$ large enough. The details of a proof parallel those of our proof of Lemma 5.7, but are simpler in the present case since $U_{g}(\lambda)=0$ when $P(\lambda, h) \neq 0$.

\section{Conclusion of the proof of Theorem 4.4}

In this section, we prove Theorem 4.4. As we have observed earlier, this theorem implies Theorem 1.4, and thus the story ends with this section.

Our proof will be accomplished by estimating the various terms in the identity

$$
\bigodot_{m, n, k}(g)=A_{m, n}(k)+B_{m, n}(k)+C_{m, n}(k)+D_{m, n}(k)+E_{m, n}(k)
$$

(see (4.6)). We start by considering the "error term"

$$
E_{m, n}(k)=\int_{\mathbb{C}^{k+1}} r(\lambda, h) R_{m, n, k+1}\left(\lambda, \lambda \mathbf{1}_{k}+h\right) \mathrm{d} A_{k+1}(\lambda, h)
$$

where $r(\lambda, h)$ is the remainder term of order 3 from Taylor's formula applied to the function $h \mapsto G_{k}\left(\lambda \mathbf{1}_{k}+h\right)$ at $h=0$ (see (4.4)). In this case we have $r(\lambda, h)=$ $G_{k}\left(\lambda \mathbf{1}_{k}+h\right)-P_{2}(\lambda, h)$, where $P_{2}$ is a polynomial of degree 2 in $h$ with the property that $P_{2}(\lambda, h)=0$ when $\lambda \notin \operatorname{supp}(g)$. It follows from Remark 5.8 that, when $m \rightarrow \infty$ and $|n-m \tau| \leq 1$ (with $Y_{m, k}$ as in (5.11)), we have

$$
\int_{\mathbb{C}^{k+1} \backslash Y_{m, k}} P_{2}(\lambda, h) R_{m, n, k+1}\left(\lambda, \lambda \mathbf{1}_{k}+h\right) \mathrm{d} A_{k+1}(\lambda, h) \rightarrow 0 .
$$

Using (6.1) and Lemma 5.7, we conclude that

$$
\int_{\mathbb{C}^{k+1} \backslash Y_{m, k}} r(\lambda, h) R_{m, n, k+1}\left(\lambda, \lambda \mathbf{1}_{k}+h\right) \mathrm{d} A_{k+1}(\lambda, h) \rightarrow 0
$$

when $m \rightarrow \infty$ and $|n-m \tau| \leq 1$. In order to estimate the integral over $Y_{m, k}$, we first introduce some notation. 
For a measurable subset $\Omega \subset \mathbb{C}^{N}$, let us denote the (suitably normalized) complex $N$-dimensional volume of $U$ by $\operatorname{Vol}_{N}(\Omega)=\int_{\Omega} \mathrm{d} A_{N}\left(\lambda_{1}, \ldots, \lambda_{N}\right)$. We write $\operatorname{Area}(\Omega)$ instead of $\operatorname{Vol}_{1}(\Omega)$.

For large $m$, the set $Y_{m, k}$ is contained in the set

$$
\left\{(\lambda, h) ; \lambda \in 8_{\tau},|h|_{\infty} \leq M k \delta_{m}\right\},
$$

whence

$$
\operatorname{Vol}_{k+1}\left(Y_{m, k}\right) \leq \operatorname{Area}\left(\delta_{\tau}\right)\left(M k \delta_{m}\right)^{2 k}=C \delta_{m}^{2 k},
$$

with $C$ a number depending on $k, M$, and $\tau$. Furthermore, (5.1) yields that

$$
\left|R_{m, n, k+1}\left(\lambda, \lambda \mathbf{1}_{k}+h\right)\right| \leq C m^{k+1}, \quad n \leq m \tau+1,
$$

for all $\lambda$ and $h$. Now, since $|r(\lambda, h)| \leq C|h|^{3} \leq C \delta_{m}^{3}$ when $|h| \leq M k \delta_{m}$, (5.1) yields

$$
\begin{aligned}
& \int_{Y_{m, k}}\left|r(\lambda, h) R_{m, n, k+1}\left(\lambda, \lambda \mathbf{1}_{k}+h\right)\right| \mathrm{d} A_{k+1}(\lambda, h) \leq C \delta_{m}^{3} m^{k+1} \operatorname{Vol}_{k+1}\left(Y_{m, k}\right) \\
& \quad=C m^{k+1} \delta_{m}^{2 k+3}=C \log ^{2 k+3} m / \sqrt{m}
\end{aligned}
$$

Hence, the integral over $Y_{m, k}$ also converges to zero when $m \rightarrow \infty$ and $|n-m \tau| \leq 1$. We have shown that $E_{m, n}(k) \rightarrow 0$ as $m \rightarrow \infty$ and $|n-m \tau| \leq 1$.

Next, we consider the term

$$
D_{m, n}(k)=\int_{\mathbb{C}^{2}}\left(\Delta_{k} G_{k}\right)\left(\lambda \mathbf{1}_{k}\right)\left|h_{1}\right|^{2} R_{m, n, 2}\left(\lambda, \lambda+h_{1}\right) \mathrm{d} A_{2}\left(\lambda, h_{1}\right) .
$$

In view of Lemma 3.3, we plainly have

$$
D_{m, n}(k)=0 \quad \text { if } k \geq 3 \text {. }
$$

It thus remains to consider the case $k=2$. In this case, Lemma 3.3 implies that

$$
D_{m, n}(2)=\frac{1}{2} \int_{\mathbb{C}^{2}}|\nabla g(\lambda)|^{2}|h|^{2} R_{m, n, 2}(\lambda, \lambda+h) \mathrm{d} A_{2}(\lambda, h) .
$$

It is clear from Remark 5.8 that

$$
\int_{|h| \geq 2 M \delta_{m}}|\nabla g(\lambda)|^{2}|h|^{2} R_{m, n, 2}(\lambda, \lambda+h) \mathrm{d} A_{2}(\lambda, h) \rightarrow 0
$$

as $m \rightarrow \infty$ and $|n-m \tau| \leq 1$. To estimate the integral over $\left\{|h| \leq 2 M \delta_{m}\right\}$, we apply the asymptotics for $R_{m, n, 2}$ from (2.3) (with the compact set $K$ replaced by supp $g$ ). It 
yields that there are numbers $v_{m}$ converging to 1 when $m \rightarrow \infty$ such that

$$
\begin{gathered}
\int_{|h| \leq 2 M \delta_{m}}|\nabla g(\lambda)|^{2}|h|^{2} R_{m, n, 2}(\lambda, \lambda+h) \mathrm{d} A_{2}(\lambda, h) \\
=v_{m} m^{2} \int_{|h| \leq 2 M \delta_{m}}|\nabla g(\lambda)|^{2}|h|^{2}\left(\Delta Q(\lambda)^{2}+\mathcal{O}\left(\delta_{m}\right)\right) \\
\quad \times e^{-m \Delta Q(\lambda)|h|^{2}} \mathrm{~d} A_{2}(\lambda, h)+o(1)
\end{gathered}
$$

when $m \rightarrow \infty$ and $n \geq m \tau-1$. Now, for a fixed $\lambda \in \operatorname{supp} g$, the change of variables $\xi=\sqrt{m \Delta Q(\lambda)} h$ shows that

$$
\int_{|h| \leq 2 M \delta_{m}}(m \Delta Q(\lambda))^{2}|h|^{2} e^{-m \Delta Q(\lambda)|h|^{2}} \mathrm{~d} A(h)=\int_{|\xi| \leq 2 M \log m}|\xi|^{2} e^{-|\xi|^{2}} \mathrm{~d} A(\xi) \rightarrow 1
$$

as $m \rightarrow \infty$. Hence, it follows from (6.2) and (6.3) that

$$
D_{m, n}(2) \rightarrow \frac{1}{2} \int_{\mathbb{C}}|\nabla g(\lambda)|^{2} \mathrm{~d} A(\lambda)
$$

as $m \rightarrow \infty$ and $|n-m \tau| \leq 1$.

The complete asymptotics for $D_{m, n}(k)$ have now been settled, and we turn to the term

$$
B_{m, n}(k)=\operatorname{Re} \int_{\mathbb{C}^{2}} S(\lambda) h^{2} R_{m, n, 2}(\lambda, \lambda+h) \mathrm{d} A_{2}(\lambda, h),
$$

where we have put

$$
S(\lambda)=\sum_{i=1}^{k}\left(\partial_{i}^{2} G_{k}\right)\left(\lambda \mathbf{1}_{k}\right)
$$

Note that supp $S \subset \operatorname{supp} g$. Using Remark 5.8, we obtain (as before) that

$$
\int_{|h| \geq 2 M \delta_{m}} S(\lambda) h^{2} R_{m, n, 2}(\lambda, \lambda+h) \mathrm{d} A_{2}(\lambda, h) \rightarrow 0
$$

as $m \rightarrow \infty$ and $|n-m \tau| \leq 1$. When $|h| \leq 2 M \delta_{m}$, we again use the asymptotics in (2.3), which yields that there are numbers $v_{m}$ converging to 1 as $m \rightarrow \infty$ such that

$$
\begin{gathered}
\int_{|h| \leq 2 M \delta_{m}} S(\lambda) h^{2} R_{m, n, 2}(\lambda, \lambda+h) \mathrm{d} A_{2}(\lambda, h) \\
=v_{m} m^{2} \int_{|h| \leq 2 M \delta_{m}} S(\lambda) h^{2}\left(\Delta Q(\lambda)^{2}+\mathcal{O}\left(\delta_{m}\right)\right) \\
\times e^{-m \Delta Q(\lambda)|h|^{2}} \mathrm{~d} A_{2}(\lambda, h)+o(1) .
\end{gathered}
$$


Now, using that, for a fixed $\lambda \in \operatorname{supp} g$,

$$
\int_{|h| \leq 2 M \delta_{m}}(m \Delta Q(\lambda))^{2} h^{2} e^{-m \Delta Q(\lambda)|h|^{2}} \mathrm{~d} A(h)=\int_{|\xi| \leq 2 M \log m} \xi^{2} e^{-|\xi|^{2}} \mathrm{~d} A(\xi)=0,
$$

we infer that $B_{m, n}(k) \rightarrow 0$ for all $k \geq 2$ as $m \rightarrow \infty$ and $|n-m \tau| \leq 1$.

It remains to estimate the terms $A_{m, n}(k)$ and $C_{m, n}(k)$. These terms are a little more complicated than the previous ones since they are defined as integrals over $\mathbb{C}^{3}$ and not over $\mathbb{C}^{2}$. We first turn to the term $A_{m, n}(k)$, which we now write in the form

$$
A_{m, n}(k)=\frac{1}{2} \int_{\mathbb{C}^{3}}\left(T(\lambda) h_{1} h_{2}+\overline{T(\lambda)} \bar{h}_{1} \bar{h}_{2}\right) R_{m, n, 3}\left(\lambda, \lambda+h_{1}, \lambda+h_{2}\right) \mathrm{d} A_{3}\left(\lambda, h_{1}, h_{2}\right),
$$

where we have put

$$
T(\lambda)=\sum_{i \neq j}\left(\partial_{i} \partial_{j} G_{k}\right)\left(\lambda \mathbf{1}_{k}\right)
$$

It is clear that supp $T \subset \operatorname{supp} g$. Furthermore, using Remark 5.8, we note as before that, with $h=\left(h_{1}, h_{2}\right)$ and $|h|_{\infty}=\max \left\{\left|h_{1}\right|,\left|h_{2}\right|\right\}$, we have

$$
\int_{|h|_{\infty} \geq 3 M \delta_{m}} \operatorname{Re}\left(T(\lambda) h_{1} h_{2}\right) R_{m, n, 3}\left(\lambda, \lambda+h_{1}, \lambda+h_{2}\right) \mathrm{d} A_{3}\left(\lambda, h_{1}, h_{2}\right) \rightarrow 0
$$

as $m \rightarrow \infty$ and $|n-m \tau| \leq 1$. When $|h|_{\infty} \leq 3 M \delta_{m}$, we insert the asymptotics for $R_{m, n, 3}$ provided by (2.4). It yields that there are numbers $v_{m}$ converging to 1 as $m \rightarrow \infty$ such that

$$
\begin{gathered}
\int_{|h|_{\infty} \leq 3 M \delta_{m}} T(\lambda) h_{1} h_{2} R_{m, n, 3}\left(\lambda, \lambda+h_{1}, \lambda+h_{2}\right) \mathrm{d} A_{3}(\lambda, h) \\
=m^{3} v_{m} \int_{|h|_{\infty} \leq 3 M \delta_{m}} T(\lambda) h_{1} h_{2}\left(\Delta Q(\lambda)^{3}+\mathcal{O}\left(\delta_{m}\right)\right) \\
\times e^{m \Delta Q(\lambda)\left(h_{1} \bar{h}_{2}-\left|h_{1}\right|^{2}-\left|h_{2}\right|^{2}\right)} \mathrm{d} A_{3}(\lambda, h)+o(1) .
\end{gathered}
$$

Now fix $\lambda \in \operatorname{supp} g$, and put $\xi_{1}=\sqrt{m \Delta Q(\lambda)} h_{1}$ and $\xi_{2}=\sqrt{m \Delta Q(\lambda)} h_{2}$. We then have that

$$
\begin{gathered}
m^{3} v_{m} \int_{|h|_{\infty} \leq 3 M \delta_{m}} T(\lambda)\left(\Delta Q(\lambda)^{3}+\mathcal{O}\left(\delta_{m}\right)\right) h_{1} h_{2} e^{m \Delta Q(\lambda)\left(h_{1} \bar{h}_{2}-\left|h_{1}\right|^{2}-\left|h_{2}\right|^{2}\right)} \mathrm{d} A_{2}(h) \\
=T(\lambda) \int_{|\xi|_{\infty} \leq 3 M \log m}\left(1+\mathcal{O}\left(\delta_{m}\right)\right) \xi_{1} \xi_{2} e^{\xi_{1} \bar{\xi}_{2}-\left|\xi_{1}\right|^{2}-\left|\xi_{2}\right|^{2}} \mathrm{~d} A_{2}(\xi) .
\end{gathered}
$$


Thus, when we can prove that $J=0$ and $J^{\prime}=0$, where $J=\int_{\mathbb{C}^{2}} \xi_{1} \xi_{2} e^{\xi_{1} \bar{\xi}_{2}-\left|\xi_{1}\right|^{2}-\left|\xi_{2}\right|^{2}} \mathrm{~d} A_{2}\left(\xi_{1}, \xi_{2}\right) \quad$ and $\quad J^{\prime}=\int_{\mathbb{C}^{2}} \bar{\xi}_{1} \bar{\xi}_{2} e^{\xi_{1} \bar{\xi}_{2}-\left|\xi_{1}\right|^{2}-\left|\xi_{2}\right|^{2}} \mathrm{~d} A_{2}\left(\xi_{1}, \xi_{2}\right)$,

we obtain the result that $A_{m, n}(k) \rightarrow 0$ as $m \rightarrow \infty$ and that $|n-m \tau| \leq 1$ for all $k \geq 2$.

The argument for $J^{\prime}$ is similar, so we settle for proving that $J=0$. To this end, we write the integral in polar coordinates

$$
J=\frac{1}{\pi^{2}} \int_{0}^{\infty} \int_{0}^{\infty} I(r, \rho) \mathrm{d} r \mathrm{~d} \rho
$$

where

$$
I(r, \rho)=\int_{0}^{2 \pi} \int_{0}^{2 \pi}(r \rho)^{2} e^{\mathrm{i}(\theta+\phi)} e^{r \rho e^{\mathrm{i}(\theta-\phi)}-r^{2}-\rho^{2}} \mathrm{~d} \phi \mathrm{d} \theta .
$$

Performing the change of variables $\vartheta=\theta+\pi / 2$ and $\varphi=\phi+\pi / 2$, the latter integral transforms to

$$
I(r, \rho)=\int_{0}^{2 \pi} \int_{0}^{2 \pi}(r \rho)^{2} e^{\mathrm{i}(\pi+\vartheta+\varphi)} e^{r \rho e^{\mathrm{i}(\vartheta-\varphi)}-r^{2}-\rho^{2}} \mathrm{~d} \vartheta \mathrm{d} \varphi=-I(r, \rho) .
$$

Hence, $I(r, \rho)=0$ for all $r$ and $\rho$, and it follows that $J=0$.

It remains to consider the term

$$
C_{m, n}(k)=\int_{\mathbb{C}^{3}}\left(Z_{k}(\lambda) h_{1} \bar{h}_{2}+\overline{Z_{k}(\lambda)} \bar{h}_{1} h_{2}\right) R_{m, n, 3}\left(\lambda, \lambda+h_{1}, \lambda+h_{2}\right) \mathrm{d} A_{3}\left(\lambda, h_{1}, h_{2}\right),
$$

where

$$
Z_{k}(\lambda)=\sum_{i<j}\left(\partial_{i} \bar{\partial}_{j} G_{k}\right)\left(\lambda \mathbf{1}_{k}\right)
$$

Observing that supp $Z_{k} \subset \operatorname{supp} g$ and arguing as in the case of $A_{m, n}(k)$, we see that

$$
\int_{|h|_{\infty} \geq 3 M \delta_{m}} Z_{k}(\lambda) h_{1} \bar{h}_{2} R_{m, n, 3}\left(\lambda, \lambda+h_{1}, \lambda+h_{2}\right) \mathrm{d} A_{3}\left(\lambda, h_{1}, h_{2}\right) \rightarrow 0
$$


as $m \rightarrow \infty$ and $|n-m \tau| \leq 1$. Hence, using (2.4), we obtain that the asymptotics of $C_{m, n}(k)$ are that of $C_{m, n}^{\prime}(k)+C_{m, n}^{\prime \prime}(k)$, where

$$
\begin{aligned}
& C_{m, n}^{\prime}(k) \\
& \quad=\int_{|h|_{\infty} \leq 3 M \delta_{m}} Z_{k}(\lambda) h_{1} \bar{h}_{2} R_{m, n, 3}\left(\lambda, \lambda+h_{1}, \lambda+h_{2}\right) \mathrm{d} A_{3}\left(\lambda, h_{1}, h_{2}\right) \\
& \quad=m^{3} v_{m} \int_{|h|_{\infty} \leq 3 M \delta_{m}} Z_{k}(\lambda) h_{1} \bar{h}_{2}\left(\Delta Q(\lambda)^{3}+\mathcal{O}\left(\delta_{m}\right)\right) e^{m \Delta Q(\lambda)\left(h_{1} \bar{h}_{2}-\left|h_{1}\right|^{2}-\left|h_{2}\right|^{2}\right)} \mathrm{d} A_{3}(\lambda, h) \\
& \quad=v_{m} \int_{\mathbb{C}} Z_{k}(\lambda)\left(\int_{|\xi|_{\infty} \leq 3 M \log m}\left(1+\mathcal{O}\left(\delta_{m}\right)\right) \xi_{1} \bar{\xi}_{2} e^{\xi_{1} \bar{\xi}_{2}-\left|\xi_{1}\right|^{2}-\left|\xi_{2}\right|^{2}} \mathrm{~d} A_{2}\left(\xi_{1}, \xi_{2}\right)\right) \mathrm{d} A(\lambda)
\end{aligned}
$$

and (likewise)

$$
\begin{aligned}
& C_{m, n}^{\prime \prime}(k) \\
& \quad=v_{m} \int_{\mathbb{C}} \overline{Z_{k}(\lambda)}\left(\int_{|\xi|_{\infty} \leq 3 M \log m}\left(1+\mathcal{O}\left(\delta_{m}\right)\right) \bar{\xi}_{1} \xi_{2} e^{\xi_{1} \bar{\xi}_{2}-\left|\xi_{1}\right|^{2}-\left|\xi_{2}\right|^{2}} \mathrm{~d} A_{2}\left(\xi_{1}, \xi_{2}\right)\right) \mathrm{d} A(\lambda),
\end{aligned}
$$

where $v_{m} \rightarrow 1$ as $m \rightarrow \infty$.

We first claim that $C_{m, n}^{\prime}(k) \rightarrow 0$ when $m \rightarrow \infty$ and $|n-m \tau| \leq 1$ for all $k \geq 2$. We will have shown this when we can prove that $L^{\prime}=0$, where

$$
L^{\prime}=\int_{\mathbb{C}^{2}} \xi_{1} \bar{\xi}_{2} e^{\xi_{1} \bar{\xi}_{2}-\left|\xi_{1}\right|^{2}-\left|\xi_{2}\right|^{2}} \mathrm{~d} A_{2}\left(\xi_{1}, \xi_{2}\right)
$$

To prove this, we pass to polar coordinates, and we write

$$
L^{\prime}=\frac{1}{\pi^{2}} \int_{0}^{\infty} \int_{0}^{\infty} P(r, \rho) \mathrm{d} r \mathrm{~d} \rho
$$

where

$$
P(r, \rho)=\int_{0}^{2 \pi} \int_{0}^{2 \pi}(r \rho)^{2} e^{\mathrm{i}(\theta-\phi)} e^{r \rho e^{\mathrm{i}(\theta-\phi)}-r^{2}-\rho^{2}} \mathrm{~d} \theta \mathrm{d} \phi .
$$

Making the change of variables $\vartheta=\theta-\phi$ and $\varphi=\phi$, the integral transforms to

$$
P(r, \rho)=e^{-r^{2}-\rho^{2}} \int_{0}^{2 \pi}\left(\int_{-\varphi}^{2 \pi-\varphi}(r \rho)^{2} e^{\mathrm{i} \vartheta} e^{r \rho e^{\mathrm{i} \vartheta}} \mathrm{d} \vartheta\right) \mathrm{d} \varphi .
$$


But the inner integral is readily calculated,giving

$$
\int_{-\varphi}^{2 \pi-\varphi}(r \rho)^{2} e^{\mathrm{i} \vartheta} e^{r \rho e^{\mathrm{i} \vartheta}} \mathrm{d} \vartheta=\left[-\mathrm{i} r \rho e^{r \rho e^{\mathrm{i} \vartheta}}\right]_{\vartheta=-\varphi}^{2 \pi-\varphi}=0 .
$$

This shows that $P(r, \rho)=0$, and consequently that $L^{\prime}=0$. It follows that $C_{m, n}^{\prime}(k) \rightarrow$ 0 as $m \rightarrow \infty$ and that $|n-m \tau| \leq 1$ for all $k \geq 2$.

To handle the term $C_{m, n}^{\prime \prime}(k)$, it becomes necessary to calculate

$$
L^{\prime \prime}=\int_{\mathbb{C}^{2}} \bar{\xi}_{1} \xi_{2} e^{\xi_{1} \bar{\xi}_{2}-\left|\xi_{1}\right|^{2}-\left|\xi_{2}\right|^{2}} \mathrm{~d} A_{2}\left(\xi_{1}, \xi_{2}\right)
$$

Again, passing to polar coordinates, we write

$$
L^{\prime \prime}=\frac{1}{\pi^{2}} \int_{0}^{\infty} \int_{0}^{\infty} W(r, \rho) \mathrm{d} r \mathrm{~d} \rho
$$

where

$$
\begin{aligned}
W(r, \rho) & =e^{-r^{2}-\rho^{2}} \int_{0}^{2 \pi} \int_{0}^{2 \pi}(r \rho)^{2} e^{\mathrm{i}(\theta-\phi)} e^{r \rho e^{\mathrm{i}(\phi-\theta)}} \mathrm{d} \phi \mathrm{d} \theta \\
& =2 \pi e^{-r^{2}-\rho^{2}} \int_{0}^{2 \pi}(r \rho)^{2} e^{-i \vartheta} e^{r \rho e^{i \vartheta}} \mathrm{d} \vartheta
\end{aligned}
$$

We now put $z=e^{\mathrm{i} \vartheta}$, and we use a simple residue argument to get

$$
W(r, \rho)=\frac{2 \pi(r \rho)^{2} e^{-r^{2}-\rho^{2}}}{\mathrm{i}} \int_{\mathbb{T}} \frac{1}{z^{2}} e^{r \rho z} \mathrm{~d} z=4 \pi^{2}(r \rho)^{3} e^{-r^{2}-\rho^{2}} .
$$

It follows that

$$
L^{\prime \prime}=4 \int_{0}^{\infty} \int_{0}^{\infty}(r \rho)^{3} e^{-r^{2}-\rho^{2}} \mathrm{~d} r \mathrm{~d} \rho=1 .
$$

For $k=2$, it now follows from (6.7), (6.6), and Lemma 3.4 that

$$
C_{m, n}^{\prime \prime}(2) \rightarrow-\int_{\mathbb{C}}|\bar{\partial} g(\lambda)|^{2} \mathrm{~d} A(\lambda)
$$

when $m \rightarrow \infty$ and $|n-m \tau| \leq 1$. On the other hand, when $k \geq 3$, we get

$$
\lim _{m \rightarrow \infty,|n-m \tau| \leq 1} C_{m, n}^{\prime \prime}(k)=\int_{\mathbb{C}} \overline{Z_{k}(\lambda)} \mathrm{d} A(\lambda),
$$

which is pure imaginary, again by Lemma 3.4. In fact, this shows that the limit in (6.8) must vanish because the cumulant $\ell_{m, n, k}(g)$ is real and because all other terms in the 
expansion (in Lemma 4.3) except $C_{m, n}(k)$ have already been shown to be real (in fact zero) in the limit when $m \rightarrow \infty$ and $|n-m \tau| \leq 1$.

The proofs of all statements are now complete.

\section{Concluding remarks}

We conclude this paper with a series of remarks concerning possible applications and generalizations of our main theorem. We also outline an alternative approach to the proof of Theorem 1.4.

\subsection{Nonanalytic potentials}

Recall that we proved Theorem 1.4 by assuming that the potential $Q$ is real analytic in some neighborhood of $\delta_{\tau}$. It is possible to extend this result to more general smooth potentials. Assuming that $Q$ is $\mathcal{C}^{\infty}$-smooth, one defines the auxiliary functions $\psi, b_{0}$, and $b_{1}$ in the expression

$$
K_{m}^{1}(z, w)=\left(m b_{0}(z, \bar{w})+b_{1}(z, \bar{w})\right) e^{m \psi(z, \bar{w})}
$$

as any fixed almost-holomorphic extensions from the antidiagonal of $Q, \Delta Q$, and $(1 / 2) \Delta \log \Delta Q$, respectively. For example, in the case of $\psi$ this means that $\psi$ is well defined and smooth in a neighborhood of the antidiagonal in $\mathbb{C}^{2}$, and that (i) $\psi(z, \bar{z})=Q(z)$, (ii) the antiholomorphic derivatives $\bar{\partial}_{i} \psi$ vanish to infinite order at each point of the antidiagonal $i=1,2$, and (iii) $\psi(z, w)=\overline{\psi(\bar{w}, \bar{z})}$ whenever the expressions make sense. Lemma 1.2 extends to this more general situation; the proof is not very different from the argument in [1], but it involves some additional technical work. The rest of the proof of Theorem 1.4 for smooth potentials requires only minor changes.

As we mentioned earlier, the smoothness (or analyticity) condition is local-we need it only in some neighborhood of the droplet. In particular, Theorem 1.4 is true for potentials $Q: \mathbb{C} \rightarrow \mathbb{R} \cup\{+\infty\}$ of the form

$$
Q(z)=Q_{0}(z)+\int_{\mathbb{C}} \log \frac{1}{\left|z-z_{0}\right|^{2}} \mathrm{~d} \mu\left(z_{0}\right),
$$

where $Q_{0}$ is a smooth function (with sufficient growth at infinity) and where $\mu$ is a positive, finitely supported measure (linear combination of Dirac measures). In this case, the droplet $\delta$ is disjoint from $\operatorname{supp} \mu$, and so the local smoothness condition holds. (We need this observation later.)

\subsection{Variational approach}

Here we sketch a different, more "physical" proof of our main result, Theorem 1.4. The proof is based on a variational argument well known in the physical literature (see, 
e.g., the papers of Wiegmann and Zabrodin). In the rigorous mathematical setting, this method was developed by Johansson in the 1-dimensional case (see [22]).

We use the fact that the estimate (1.12) for $K_{m, n}(z, z) e^{-m Q(z)}$ is uniform when we make small smooth perturbations of the potential $Q$. We also need some basic facts concerning the variation of the droplet under the change of potential (Hele-Shaw theory). Modulo these technical issues (see Remark 7.2.1), the proof of the theorem is rather short.

To simplify the notation, we assume that $m=n$ and $\tau=1$, and we write $K_{n}$ instead of $K_{n, n}$, and so forth. Let $h: \mathbb{C} \rightarrow \mathbb{R}$ be a bounded smooth function. We denote, for a positive integer $n$,

$$
Q_{n}(z)=Q(z)-\frac{h(z)}{n},
$$

and we use "tilde notation" for various objects defined with respect to the weight $Q_{n}$. Thus $\widetilde{K}_{n}$ is the kernel function with respect to $Q_{n}$ and so on, while the usual notation ( $K_{n}$, and so forth) is reserved for the weight $Q$.

It is known that for any $K \Subset \mathcal{8}_{1}^{\circ} \cap X$, the coincidence set $\left\{Q_{n}=\left(\widehat{Q}_{n}\right)_{1}\right\}$, and therefore the perturbed droplet, contains $K$ in its interior when $n$ is large enough. One can then prove that

$$
\widetilde{K}_{n}(z, z) e^{-n Q_{n}(z)}=n \Delta Q_{n}(z)+\frac{1}{2} \Delta \log \Delta Q_{n}(z)+o(1), \quad(n \rightarrow \infty),
$$

for $z \in K$ and that the $o(1)$-term is uniform in $z$.

Let $g \in \mathcal{C}_{0}^{\infty}\left(\wp_{1}^{\circ} \cap X\right)$. Therefore, we have

$$
\widetilde{K}_{n}(z, z) e^{-n Q_{n}(z)}=n \Delta Q(z)-\Delta h(z)+\frac{1}{2} \Delta \log \Delta Q(z)+o(1)
$$

uniformly for $z \in \operatorname{supp} g$. We define

$$
D_{n}^{h}[g]=\widetilde{E}_{n}\left(\text { fluct }_{n} g\right) .
$$

If $V$ denotes the Vandermonde determinant, we then have (see (1.3) and (1.2))

$$
\begin{aligned}
& D_{n}^{h}[g]=\frac{\int_{\mathbb{C}^{n}} \text { fluct }_{n} g \cdot|V|^{2} e^{-n \text { trace }_{n} Q_{n}} \mathrm{~d} A_{n}}{\int_{\mathbb{C}^{n}}|V|^{2} e^{-n} \text { trace }_{n} Q_{n} \mathrm{~d} A_{n}} \\
& =\frac{\int_{\mathbb{C}^{n}} \text { fluct }_{n} g \cdot e^{\text {trace }_{n} h}|V|^{2} e^{-n \operatorname{trace}_{n} Q} \mathrm{~d} A_{n}}{\int_{\mathbb{C}^{n}} e^{\text {trace }_{n} h}|V|^{2} e^{-n \operatorname{trace}_{n} Q} \mathrm{~d} A_{n}}=\frac{E_{n}\left(\text { fluct }_{n} g \cdot e^{\text {trace }_{n} h}\right)}{E_{n}\left(e^{\text {trace }_{n} h}\right)} .
\end{aligned}
$$

We now fix a real-valued $g$, and we set

$$
h=\lambda\left(g-\int g \Delta Q \mathrm{~d} A\right),
$$


where $\lambda$ is a real number, so that

$$
\text { trace }_{n} h=\lambda \text { fluct }_{n} g .
$$

We have

$$
\begin{gathered}
D_{n}^{h}[g]=\frac{E_{n}\left(\text { fluct }_{n} g \cdot e^{\lambda \text { fluct }_{n} g}\right)}{E_{n}\left(e^{\lambda \text { fluct }_{n} g}\right)}=F_{n}^{\prime}(\lambda), \\
\text { where } F_{n}(\lambda):=\log \left(E_{n} e^{\lambda \text { fluct }_{n} g}\right) .
\end{gathered}
$$

Now, from (7.1), we see that

$$
\begin{aligned}
D_{n}^{h}[g] & =\int_{\mathbb{C}} g(z) \widetilde{K}_{n}(z, z) e^{-n q_{n}(z)} \mathrm{d} A(z)-n \int_{\mathbb{C}} g \Delta Q \mathrm{~d} A \\
& =-\int \Delta h \cdot g \mathrm{~d} A+\int g \mathrm{~d} \nu+o(1) \rightarrow \int \partial h \cdot \bar{\partial} g \mathrm{~d} A+\int g \mathrm{~d} \nu .
\end{aligned}
$$

It follows from (7.2) that

$$
F_{n}^{\prime}(\lambda) \rightarrow \int g \mathrm{~d} \nu+\frac{\lambda}{4} \int|\nabla g|^{2} \mathrm{~d} A \quad \text { as } n \rightarrow \infty .
$$

The last relation can be integrated over $\lambda \in[0,1]$. This is justified by dominated convergence and the estimate $F_{n}^{\prime \prime} \geq 0$, which is just the Cauchy-Schwarz inequality. It follows that

$$
\log E_{n} e^{\text {fluct }_{n} g}=F_{n}(1)=\int_{0}^{1} F_{n}^{\prime}(\lambda) \mathrm{d} \lambda \rightarrow \int g \mathrm{~d} \nu+\frac{1}{8} \int|\nabla g|^{2} \mathrm{~d} A
$$

when $n \rightarrow \infty$. This means that

$$
\log E_{n} e^{t \text { fluct }_{n} g} \rightarrow t e_{g}+t^{2} v_{g}^{2} / 2
$$

for all suitable scalars $t$, which in turn implies Theorem 1.4.

\section{Remark 7.2.1}

We have discussed two rather different approaches to our main result Theorem 1.4. We note that, in the (most interesting) case when the potential is real analytic in a neighborhood of the droplet, the theory of asymptotic expansions for the correlation kernel is somewhat simpler and cleaner than in the smooth case. In the variational proof we need to make a smooth perturbation of the potential, and so we need a discussion of the smooth theory even in cases when the potential is real analytic. One would also need to include a further discussion of the Hele-Shaw theory to make the 
variational proof complete. We will discuss the variational approach in greater detail in our forthcoming paper [2].

\subsection{Interpretation in terms of Gaussian fields}

Denote $U=\vartheta_{1}^{\circ} \cap X$, and let $\mathcal{W}_{0}(U)=W_{0}^{1,2}(U)$ be the completion of $\mathcal{C}_{0}^{\infty}(U)$ under the Dirichlet inner product

$$
\langle f, g\rangle_{\nabla}=\int_{\mathbb{C}} \nabla f \cdot \overline{\nabla g} \mathrm{~d} A
$$

Let $G$ be Green's function for $U$, and denote by $\mathcal{E}(U)=W^{-1,2}(U)$ the Hilbert space of distributions with inner product

$$
\left\langle\rho_{1}, \rho_{2}\right\rangle_{\mathcal{E}}=\int_{U} \int_{U} G(z, w) \mathrm{d} \rho_{1}(z) \mathrm{d} \bar{\rho}_{2}(w) .
$$

(More accurately, $\mathcal{E}(U)$ is the completion of the space of measures with finite $\mathbb{E}$-norm.) We have an isomorphism

$$
\Delta_{U}: \mathcal{W}_{0}(U) \rightarrow \mathcal{E}(U)
$$

where $\Delta_{U}=\partial \bar{\partial}$ is the (Dirichlet) Laplacian. The inverse map is given by the Green potential

$$
-\frac{1}{2} \Delta_{U}^{-1} \rho=U_{G}^{\rho}
$$

where

$$
U_{G}^{\rho}(z)=\int_{U} G(z, w) \mathrm{d} \rho(w)
$$

By a Gaussian field indexed by $\mathcal{W}_{0}(U)$ we mean an isometry

$$
\Gamma: \mathcal{W}_{0}(U) \rightarrow L^{2}(\Omega, P),
$$

where $(\Omega, P)$ is some probability space and where $\Gamma(g) \sim N\left(0,\|g\|_{\nabla}^{2}\right)$ for any $g \in \mathcal{W}_{0}(U)$. We now pick $\left(\lambda_{j}\right)_{1}^{n}$ randomly with respect to $\Pi_{n, n}$, and we consider the sequence of random fields (measures)

$$
\Gamma_{n}=4\left(\sum_{j=1}^{n} \delta_{\lambda_{j}}-n \sigma_{1}-v\right),
$$

which satisfy

$$
\Gamma_{n}(g)=4\left(\text { fluct }_{n} g-\int g \mathrm{~d} v\right)
$$


Thus, Theorem 1.4 implies that as $n \rightarrow \infty$, the fields $\Gamma_{n}$ converge to a Gaussian field $\Gamma$ indexed by $\mathcal{W}_{0}(U)$. The precise meaning of the field convergence is convergence of the correlation functions

$$
E_{n}\left(\Gamma_{n}\left(g_{1}\right) \cdots \Gamma_{n}\left(g_{k}\right)\right) \rightarrow\left\langle\Gamma\left(g_{1}\right) \cdots \Gamma\left(g_{k}\right)\right\rangle
$$

for all finite collections of test functions $\left\{g_{j}\right\} \subset \mathcal{C}_{0}^{\infty}(U)$. The right-hand side of (7.3) is given by Wick's formulas

$$
\left\langle\Gamma\left(g_{1}\right) \cdots \Gamma\left(g_{2 p+1}\right)\right\rangle=0
$$

and

$$
\left\langle\Gamma\left(g_{1}\right) \cdots \Gamma\left(g_{2 p}\right)\right\rangle=\sum \prod_{k=1}^{p}\left\langle g_{i_{k}}, g_{j_{k}}\right\rangle_{\nabla},
$$

where the sum is over all partitions of $\{1, \ldots, 2 p\}$ into $p$ disjoint pairs $\left(i_{k}, j_{k}\right)$.

Using the identifications mentioned above, we obtain the following result.

PROPOSITION 7.3.1

The random functions

$$
h_{n}(z)=2\left(\sum_{j=1}^{n} G\left(z, \lambda_{j}\right)-U_{G}^{n \sigma_{1}+v}(z)\right)
$$

converge in $U$ to a Gaussian free field with Dirichlet boundary condition, that is, to a Gaussian field indexed by $\mathcal{E}(U)$.

Alternatively, if we pick $\left(\lambda_{j}\right)$ and $\left(\lambda_{j}^{\prime}\right)$ independently with respect to $\Pi_{n, n}$, then the random functions

$$
\tilde{h}_{n}(z)=\sum_{j=1}^{n}\left(G\left(z, \lambda_{j}\right)-G\left(z, \lambda_{j}^{\prime}\right)\right)
$$

converge to a Gaussian free field with Dirichlet boundary condition.

\subsection{Fluctuations near the boundary}

In a separate publication [2], we prove a version of Theorem 1.4 valid for general test functions which are not necessarily supported in the droplet, but just, say, of class $e_{0}^{\infty}(\mathbb{C})$. The proof is based on Ward's identities and Johansson's variational technique mentioned above. Here we settle for stating only the result.

We assume throughout that $Q$ is real analytic and strictly subharmonic in some neighborhood of the droplet $\delta=\delta_{1}$. One can then prove that the boundary $\partial \&$ is 
regular, that is, a finite union of real analytic curves. We write $\mathrm{d} s$ for the arc length measure on $\partial \ell_{1}$ divided by $2 \pi$. Denote

$$
U=8^{\circ} \quad \text { and } \quad U_{*}=\mathbb{C} \backslash \rho .
$$

We then have an orthogonal decomposition of the Sobolev space $\mathcal{W}=W^{1,2}(\mathbb{C})$

$$
\mathcal{W}=\mathcal{W}_{0}(U) \oplus \mathcal{W}(\partial \&) \oplus \mathcal{W}_{0}\left(U_{*}\right) .
$$

Here, $\mathcal{W}_{0}(U)$ and $\mathcal{W}_{0}\left(U_{*}\right)$ are identified with the subspaces of functions which are (quasi-everywhere) zero in the complement of $U$ and $U_{*}$, respectively, while the subspace $\mathcal{W}(\partial \&)$ consists of the functions which are harmonic off $\partial \&$. The orthogonal projection of $\mathcal{W}$ onto $\mathcal{W}(\partial \&)$

$$
f \mapsto f^{\partial \mathcal{S}}
$$

is just the composition of the restriction operator $\left.f \mapsto f\right|_{\partial s}$ and the operation of harmonic extension to $U \cup U_{*} \cup\{\infty\}$. For $f \in \mathcal{W}$, we also denote by $f^{s}$ the orthogonal projection of $f$ onto $\mathcal{W}_{0}(U) \oplus \mathcal{W}(\partial \&)$

$$
f^{\S}=\mathbf{1}_{s} \cdot f+\mathbf{1}_{U_{*}} \cdot f^{\text {ว }} .
$$

In other words, $f^{\delta}$ coincides with $f$ on $\delta$ and is harmonic and bounded in the complement of that set.

Finally, we write $n_{U} f$ for the exterior normal derivative of $\left.f\right|_{\delta}$, and we write $n_{U_{*}} f$ for the exterior normal derivative of $\left.f^{\partial \&}\right|_{U_{*}}$. We can now state the theorem.

\section{THEOREM 7.4.1}

Let $f \in \mathcal{C}_{0}^{\infty}(\mathbb{C})$. Then the random variables fluct $f$ on the space $\left(\mathbb{C}^{n}, \Pi_{n, n}\right)$ converge in distribution to $N\left(e_{f}, v_{f}^{2}\right)$, where

$$
v_{f}^{2}=\frac{1}{4} \int\left|\nabla\left(f^{8}\right)\right|^{2} \mathrm{~d} A
$$

and where

$$
\begin{aligned}
e_{f}= & \int_{\delta} f \mathrm{~d} v+\frac{1}{4} \int_{\partial \delta} n_{U}(f) \mathrm{d} s \\
& +\frac{1}{4} \int_{\partial \delta}\left(f \cdot n_{U_{*}}(\log \Delta Q)-n_{U_{*}}\left(f^{\partial \delta}\right) \cdot \log \Delta Q\right) \mathrm{d} s .
\end{aligned}
$$

Note that the formula for $e_{f}$ becomes very simple in the case of the so-called HeleShaw potentials, that is, if $\Delta Q=$ constant $>0$ in a neighborhood of 8 , then

$$
e_{f}=\frac{1}{4} \int_{\partial \delta} n_{U}(f) \mathrm{d} s .
$$


In field theoretical terms, Theorem 7.4.1 means that there exists a deterministic distribution $u$, given by the right-hand side of (7.4), such that the random distributions

$$
4\left(\sum_{j=1}^{n} \delta_{\lambda_{j}}-n \sigma_{1}-u\right)
$$

converge in $\mathbb{C}$ to the sum of two independent Gaussian fields indexed by $\mathcal{W}_{0}(U)$ and $\mathcal{W}\left(\partial \oiint_{1}\right)$, respectively. While the first one is conformally invariant, the second one is not.

Alternatively, we can say that the random functions

$$
h_{n}(z)=\log \left|\frac{p\left(z ; M_{1}\right)}{p\left(z ; M_{2}\right)}\right|,
$$

where the $p\left(z ; M_{j}\right)$ are the characteristic polynomials of two independent $n \times n$ random normal matrices $M_{j}$, converge to a free Gaussian field on $\&$ with free boundary condition.

\subsection{Large volume limit}

Let us take a point $z_{0} \in 8_{1}^{\circ} \cap X$ and assume for simplicity that $\Delta Q\left(z_{0}\right)=1$. Define $\mu_{n} \in \operatorname{Prob}\left(\mathbb{C}^{n}\right)$ as the image of $\Pi_{n, n}$ under the map

$$
\left(\lambda_{j}\right)_{j=1}^{n} \mapsto\left(\sqrt{n}\left(\lambda_{j}-z_{0}\right)\right)_{j=1}^{n},
$$

and think of $\mu_{n}$ as a point process in $\mathbb{C}$.

PROPOSITION 7.5.1

The processes $\mu_{n}$ converge to the Ginibre $(\infty)$ point process, that is, to the determinantal process with correlation kernel

$$
K(z, w)=e^{z \bar{w}-\left(|z|^{2}+|w|^{2}\right) / 2} .
$$

\section{Proof}

Assume without loss of generality that $z_{0}=0$. Then $\mu_{n}$ are determinantal processes with correlation kernels

$$
k_{n}(z, w)=\frac{1}{n} K_{n, n}\left(\frac{z}{\sqrt{n}}, \frac{w}{\sqrt{n}}\right) e^{-n(Q(z / \sqrt{n})+Q(w / \sqrt{n})) / 2} .
$$

Using the expansion for $K_{n, n}$ in Lemma 1.2, we see that

$$
k_{n}(z, w)=(\Delta Q(0)+o(1)) e^{n \psi(z / \sqrt{n}, \bar{w} / \sqrt{n})-n(Q(z / \sqrt{n})+Q(w / \sqrt{n})) / 2},
$$


where the $o(1)$ is uniform for $z$ and $w$ in a fixed compact subset of $\mathbb{C}$. Next, observe that, up to negligible terms, we have

$$
\psi(z, \bar{w})=Q(0)+a z+\bar{a} \bar{w}+b z^{2}+\bar{b} \bar{w}^{2}+z \bar{w}
$$

for some complex numbers $a$ and $b$. It follows that

$$
k_{n}(z, w)=(1+o(1)) e^{\mathrm{i} \sqrt{n} \operatorname{Im}(a(z-w))} e^{\mathrm{i} \operatorname{Im}\left(b\left(z^{2}-w^{2}\right)\right)} e^{z \bar{w}-\left(|z|^{2}+|w|^{2}\right) / 2} .
$$

The first two exponential factors cancel out when we compute the determinants representing intensity $k$-point functions, which yields the desired result.

\subsection{Berezin transform and fluctuations of eigenvalues}

We write

$$
R_{n}^{k}\left(\lambda_{1}, \ldots, \lambda_{k}\right)=\operatorname{det}\left(K_{n}\left(\lambda_{i}, \lambda_{j}\right)\right)_{i, j=1}^{k} e^{-n \sum_{j=1}^{k} Q\left(\lambda_{j}\right)}
$$

for the $k$-point intensity function of the ensemble (1.2) with $m=n$. We also need the connected two-point function

$$
R_{n}^{2, c}(z, w)=R_{n}^{2}(z, w)-R_{n}^{1}(z) R_{n}^{1}(w)=-\left|K_{n}(z, w)\right|^{2} e^{-n(Q(z)+Q(w))} .
$$

It is easy to check that

$$
\int_{\mathbb{C}} R_{n}^{2, c}(z, w) \mathrm{d} A(w)=-R_{n}^{1}(z)
$$

and that

$\operatorname{Cov}\left(\right.$ fluct $_{n} f$, fluct $\left._{n} g\right)=\int_{\mathbb{C}} f(z) g(z) R_{n}^{1}(z) \mathrm{d} A(z)+\int_{\mathbb{C}^{2}} f(z) g(w) R_{n}^{2, c}(z, w) \mathrm{d} A_{2}(z, w)$.

Recall that for a given $z$, the corresponding Berezin kernel $\mathrm{S}_{n}^{\langle z\rangle}$ is given by

$$
\mathrm{Б}_{n}^{\langle z\rangle}(w)=-\frac{R_{n}^{2, c}(z, w)}{R_{n}^{1}(z)}=R_{n}^{1}(w)-\frac{R_{n}^{2}(z, w)}{R_{n}^{1}(z)}
$$

and that the Berezin transform is

$$
\mathcal{B}_{n} f(z)=\int_{\mathbb{C}} f(w) \mathrm{S}_{n}^{\langle z\rangle}(w) \mathrm{d} A(w)
$$

We may now conclude that

$$
\operatorname{Cov}\left(\text { fluct }_{n} f, \text { fluct }_{n} g\right)=\int_{\mathbb{C}}\left(f(z)-\mathscr{B}_{n} f(z)\right) g(z) R_{n}^{1}(z) \mathrm{d} A(z) .
$$


On the other hand, Theorem 1.4 implies that

$$
\operatorname{Cov}\left(\text { fluct }_{n} f, \text { fluct }_{n} g\right) \rightarrow-\int_{\mathbb{C}} \Delta f(z) g(z) \mathrm{d} A(z), \quad(n \rightarrow \infty),
$$

where $f, g \in \mathcal{C}_{0}^{\infty}\left(\wp_{1}^{\circ} \cap X\right)$. Therefore, we have

$$
\int\left(f(z)-\mathscr{B}_{n} f(z)\right) R_{n}^{1}(z) g(z) \mathrm{d} A(z) \rightarrow-\int \Delta f(z) g(z) \mathrm{d} A(z) .
$$

Since

$$
R_{n}^{1}=n \Delta Q+\frac{1}{2} \Delta \log \Delta Q+o(1)
$$

on the support of $g$, we obtain the following asymptotic formula for the Berezin transform.

PROPOSITION 7.6.1

If $f \in \mathcal{C}_{0}^{\infty}\left(\wp_{1}^{\circ} \cap X\right)$, then

$$
\mathfrak{B}_{n} f=f+\frac{\Delta f}{n \Delta Q}+o\left(\frac{1}{n}\right)
$$

inside the droplet in the sense of distributions.

Berezin's transform has the following probabilistic interpretation. Let us think of the measure $\Pi_{n}=\Pi_{n, n}$ as the law of a point process $\Phi_{n}$ in $\mathbb{C}$. We refer to $\Phi_{n}$ as the $n$-point random normal matrix (RNM) process associated with potential $Q$.

Let us now condition $\Phi_{n}$ on the event $\left\{z_{0} \in \Phi_{n}\right\}$ and write $\widetilde{\Phi}_{n-1}^{\left\langle z_{0}\right\rangle}$ for conditional $(n-1)$-point process. Accordingly, we write $R_{n}^{k}$ for the $k$-point intensity function of $\Phi_{n}$, and we write $\widetilde{R}_{n-1}^{k}=\widetilde{R}_{n-1}^{k,\left\langle z_{0}\right\rangle}$ for the $k$-point function of $\widetilde{\Phi}_{n-1}^{\left\langle z_{0}\right\rangle}$.

LEMMA 7.6.2

We have

$$
\mathrm{Б}_{n}^{\left\langle z_{0}\right\rangle}(z)=R_{n}^{1}(z)-\widetilde{R}_{n-1}^{1}(z)
$$

\section{Proof}

Consider small disks $D$ and $D_{0}$ centered at $z$ and $z_{0}$ with radii $\varepsilon$ and $\varepsilon_{0}$, respectively. We have

$$
R_{n}^{1}\left(z_{0}\right)=\lim _{\varepsilon_{0} \rightarrow 0} \frac{\Pi_{n}\left(\left\{\Phi_{n} \cap D_{0} \neq \emptyset\right\}\right)}{\varepsilon_{0}^{2}}
$$


and

$$
R_{n}^{2}\left(z_{0}, z\right)=\lim _{\varepsilon, \varepsilon_{0} \rightarrow 0} \frac{\Pi_{n}\left(\left\{\Phi_{n} \cap D \neq \emptyset\right\} \cap\left\{\Phi_{n} \cap D_{0} \neq \emptyset\right\}\right)}{\varepsilon^{2} \varepsilon_{0}^{2}} .
$$

It follows that

$$
\begin{aligned}
\widetilde{R}_{n-1}^{1}(z) & =\lim _{\varepsilon \rightarrow 0} \lim _{\varepsilon_{0} \rightarrow 0} \frac{\Pi_{n}\left(\left\{\Phi_{n} \cap D \neq \emptyset \mid \Phi_{n} \cap D_{0} \neq \emptyset\right\}\right)}{\varepsilon^{2}} \\
& =\lim _{\varepsilon \rightarrow 0} \lim _{\varepsilon_{0} \rightarrow 0} \frac{\Pi_{n}\left(\left\{\Phi_{n} \cap D \neq \emptyset\right\} \cap\left\{\Phi_{n} \cap D_{0} \neq \emptyset\right\}\right)}{\varepsilon^{2} \Pi_{n}\left(\left\{\Phi_{n} \cap D_{0} \neq \emptyset\right\}\right)}= \\
& =\frac{R_{n}^{2}\left(z_{0}, z\right)}{R_{n}^{1}\left(z_{0}\right)}=R_{n}^{1}(z)-\mathrm{S}_{n}^{\left\langle z_{0}\right\rangle}(z) .
\end{aligned}
$$

Integrating (7.7) against test functions, we get the following formula, where $E_{n}$ stands for the expectation with respect to $\Pi_{n}$ and where $\widetilde{E}_{n-1}^{\left\langle z_{0}\right\rangle}$ is the expectation with respect to the law of $\widetilde{\Phi}_{n-1}^{\left\langle z_{0}\right\rangle}$.

COROLLARY 7.6.3

Let $z_{0} \in \mathbb{C}$, and let $f \in \mathcal{C}_{b}(\mathbb{C})$. Then

$$
\mathcal{B}_{n} f\left(z_{0}\right)=E_{n}\left(\operatorname{trace}_{n} f\right)-\widetilde{E}_{n-1}^{\left\langle z_{0}\right\rangle}\left(\operatorname{trace}_{n-1} f\right) .
$$

The central limit theorem for the Berezin transform states that the rescaled (as in the large volume limit procedure) Berezin measures converge to the standard Gaussian distribution in $\mathbb{C}$ (see [1, Theorem 2.6]). We can now interpret this statement in terms of random eigenvalues.

Let $z_{0} \in \mathcal{S}_{1}^{\circ} \cap X$, and assume without loss of generality that $\Delta Q\left(z_{0}\right)=1$. Define $\widehat{\Phi}_{n-1}^{\left\langle z_{0}\right\rangle}$ as a point process in $\mathbb{C}$ obtained from $\widetilde{\Phi}_{n-1}^{\left\langle z_{0}\right\rangle}$ by dilating all distances to $z_{0}$ by a factor of $\sqrt{n}$ as in Section 7.5. In other words, we condition $\Phi_{n}$ on the event " $z_{0}$ is an eigenvalue," and we rescale the distances.

PROPOSITION 7.6.4

The limiting point process of $\widehat{\Phi}_{n}^{\left\langle z_{0}\right\rangle},(n \rightarrow \infty)$, has the following 1-point intensity function:

$$
\widehat{R}^{1,\left\langle z_{0}\right\rangle}(z)=1-e^{-\left|z-z_{0}\right|^{2}}
$$


Proof

Let $\widehat{R}_{n-1}^{1,\left\langle z_{0}\right\rangle}$ denote the 1-point function of $\widehat{\Phi}_{n-1}^{\left\langle z_{0}\right\rangle}$. Similarly, let $\widehat{R}_{n}^{1}$ be the 1-point function for the process $\widehat{\Phi}_{n}$, by which we mean $\Phi_{n}$ dilated by a factor of $\sqrt{n}$ about $z_{0}$. By Proposition 7.5.1, the point processes $\widehat{\Phi}_{n}$ converge to the Ginibre $(\infty)$ point field as $n \rightarrow \infty$. The 1-point function of $\operatorname{Ginibre}(\infty)$ is $\widehat{R}^{1}(z) \equiv 1$ and its Berezin kernel is $\widehat{\mathrm{b}}^{\left\langle z_{0}\right\rangle}(z)=e^{-\left|z-z_{0}\right|^{2}}$. Conditioning equation (7.7) on the event " $z_{0}$ is an eigenvalue," we get

$$
\widehat{\mathrm{S}}_{n}^{\left\langle z_{0}\right\rangle}(z)=\widehat{R}_{n}^{1}(z)-\widehat{R}_{n-1}^{1,\left\langle z_{0}\right\rangle}(z)
$$

and by sending $n \rightarrow \infty$, we get the stated formula.

\subsection{Berezin transform in quasi-classical limit and orthogonal polynomials}

As before, let $\Phi_{n}$ be the $n$-point RNM process associated with potential $Q$. We fix a point $z_{0}$ and condition $\Phi_{n}$ on the event $\left\{z_{0} \in \Phi_{n}\right\}$.

LEMMA 7.7.1

The conditional $(n-1)$-point process $\widetilde{\Phi}_{n-1}^{\left\langle z_{0}\right\rangle}$ is the RNM process associated with the potential

$$
\widetilde{Q}(z)=Q(z)-\frac{1}{n-1}\left(\log \left|z-z_{0}\right|^{2}-Q(z)\right) .
$$

\section{Proof}

The density of the measure $\Pi_{n}$ is given by

$$
\rho\left(\lambda_{1}, \ldots, \lambda_{n}\right)=\frac{1}{Z}\left|V_{n}\left(\lambda_{1}, \ldots, \lambda_{n}\right)\right|^{2} e^{-n\left(Q\left(\lambda_{1}\right)+\cdots+Q\left(\lambda_{n}\right)\right)},
$$

where $Z$ is the normalizing factor (partition function) and $V_{n}$ the Vandermonde determinant (see (1.3)). Setting $z_{0}=\lambda_{n}$, we have

$$
\begin{aligned}
\rho\left(\lambda_{1}, \ldots, \lambda_{n-1}, z_{0}\right) & =\frac{e^{-n Q\left(z_{0}\right)}}{Z}\left|V_{n-1}\left(\lambda_{1}, \ldots, \lambda_{n-1}\right)\right|^{2} e^{-n\left(Q\left(\lambda_{1}\right)+\ldots+Q\left(\lambda_{n-1}\right)\right)+\sum_{j=1}^{n-1} \log \left|\lambda_{j}-z_{0}\right|^{2}} \\
& =\frac{e^{-n Q\left(z_{0}\right)}}{Z}\left|V_{n-1}\left(\lambda_{1}, \ldots, \lambda_{n-1}\right)\right|^{2} e^{-(n-1)\left(\widetilde{Q}_{n}\left(\lambda_{1}\right)+\cdots+\widetilde{Q}\left(\lambda_{n-1}\right)\right)} .
\end{aligned}
$$

It follows that the density of the conditional point process $\widetilde{\Phi}_{n-1}^{\left\langle z_{0}\right\rangle}$ is

$$
\tilde{\rho}\left(\lambda_{1}, \ldots, \lambda_{n-1}\right)=\frac{1}{\widetilde{Z}}\left|V_{n-1}\left(\lambda_{1}, \ldots, \lambda_{n-1}\right)\right|^{2} e^{-(n-1)\left(\widetilde{Q}_{n}\left(\lambda_{1}\right)+\cdots+\widetilde{Q}_{n}\left(\lambda_{n-1}\right)\right)},
$$

where $\widetilde{Z}$ is the corresponding normalizing factor. 
Let us now assume that the potential $Q$ is real analytic and strictly subharmonic in some neighborhood of the droplet $\delta=\wp_{1}$ so that Theorem 7.4.1 applies. Denote

$$
\widetilde{Q}_{n}(z)=Q(z)-\frac{h(z)}{n}, \quad h(z):=\log \left|z-z_{0}\right|^{2}-Q(z),
$$

that is, so that $\widetilde{Q}_{n}=Q-h / n$. As in Section 7.2, for a bounded smooth function $f$, we write

$$
D_{n}[f]=E_{n}\left(\text { fluct }_{n} f\right), \quad D_{n}^{h}[f]=\widetilde{E}_{n}\left(\text { fluct }_{n} f\right),
$$

where $\widetilde{E}_{n}$ is the expectation with respect to the potential $\widetilde{Q}_{n}$.

The argument in Section 7.2 shows that the variance part of Theorem 7.4.1 is equivalent to the statement that

$$
D_{n}[f]-D_{n}^{h}[f] \rightarrow \frac{1}{4}\left\langle f^{\S}, h\right\rangle_{\nabla}
$$

where $f^{\mathcal{S}}$ is the orthogonal projection of $f$ onto $\mathcal{W}_{0}(U) \oplus \mathcal{W}(\partial \&)$. By Corollary 7.6.3 and Lemma 7.7.1, we have

$$
\begin{aligned}
\mathcal{B}_{n} f\left(z_{0}\right) & =E_{n}\left(\operatorname{trace}_{n} f\right)-\widetilde{E}_{n-1}\left(\operatorname{trace}_{n-1} f\right) \\
& =\int f \mathrm{~d} \sigma+E_{n}\left(\text { fluct }_{n} f\right)-E_{n-1}\left(\text { fluct }_{n-1} f\right) \\
& =\int f \mathrm{~d} \sigma+D_{n}[f]-D_{n-1}^{h}[f]
\end{aligned}
$$

and therefore

$$
\mathcal{B}_{n} f\left(z_{0}\right) \rightarrow \int f^{\S} \mathrm{d} \sigma+\left\langle f^{\S}, h\right\rangle_{\nabla}, \quad(n \rightarrow \infty)
$$

Note that

$$
\left\langle f^{s}, h\right\rangle_{\nabla}=\left\langle f^{s}, Q^{s}\right\rangle_{\nabla}-\left\langle f^{s}, l\right\rangle_{\nabla}
$$

where $l(z)=\log \left|z-z_{0}\right|^{2}$. Also, we have

$$
\left\langle f^{\S}, Q^{\S}\right\rangle_{\nabla}=-\int f^{\S} \Delta Q^{\S} \mathrm{d} A=-\int f \mathrm{~d} \sigma
$$

and

$$
-\left\langle f^{\S}, l\right\rangle_{\nabla}=\int f^{s} \Delta l \mathrm{~d} A=f^{\S}\left(z_{0}\right)
$$


In view of (7.10), it follows that

$$
\mathscr{B}_{n} f\left(z_{0}\right) \rightarrow f^{\mathcal{S}}\left(z_{0}\right)
$$

Since the function $f$ was arbitrary, we have derived the following result.

THEOREM 7.7.2

Let $z_{0} \in \mathbb{C}$. Then the Berezin measures $B_{n}^{\left\langle z_{0}\right\rangle} \mathrm{d} A$ converge to the Dirac measure at $z_{0}$ if $z_{0} \in \delta_{1}$, and to the harmonic measure of $\mathbb{C} \backslash \delta_{1}$ evaluated at $z_{0}$ if $z_{0} \notin \boldsymbol{s}_{1}$.

This theorem is also true at $z_{0}=\infty$, in which case it has the following form.

\section{THEOREM 7.7.3}

Let $P_{n}$ be the $n$th orthonormal polynomial with respect to the measure $e^{-n Q} \mathrm{~d} A$ in $\mathbb{C}$. Then the probability measures

$$
\left|P_{n}\right|^{2} e^{-n Q} \mathrm{~d} A
$$

converge to the harmonic measure of $\widehat{\mathbb{C}} \backslash \wp_{1}$ evaluated at $\infty$.

Proof

We need to compute the limit of the Berezin kernel $\mathrm{S}_{n}^{\left\langle z_{0}\right\rangle}(z)$ as $z_{0} \rightarrow \infty$. By Lemma 7.7, we have

$$
\mathrm{Б}_{n}^{\left\langle z_{0}\right\rangle}(z)=R_{n}^{1}(z)-\widetilde{R}_{n-1}^{1}(z)
$$

where $R_{n}^{1}$ and $\widetilde{R}_{n-1}^{1}$ are the 1-point functions of $\Phi_{n}$ and $\widetilde{\Phi}_{n-1}^{\left\langle z_{0}\right\rangle}$, respectively. Since $\Phi_{n}$ is the $n$-point RNM process associated with potential $Q$, we have

$$
R_{n}^{1}=\sum_{k=0}^{n-1}\left|P_{k}\right|^{2} e^{-n Q}
$$

On the other hand, by Lemma 7.7.1, $\widetilde{\Phi}_{n-1}^{\left\langle z_{0}\right\rangle}$ is the $(n-1)$-point RNM process associated with the potential

$$
\widetilde{Q}^{\left\langle z_{0}\right\rangle}(z)=\frac{n}{n-1} Q(z)+\frac{1}{n-1} \log \left(\frac{\left|z_{0}\right|^{2}}{\left|z-z_{0}\right|^{2}}\right) .
$$

(Here we added a constant term to the potential $\widetilde{Q}$ in Lemma 7.7.1; this clearly did not affect the point process.) Since

$$
\widetilde{Q}^{\left\langle z_{0}\right\rangle}(z) \rightarrow \widetilde{Q}(z):=\frac{n}{n-1} Q(z) \quad \text { as } z_{0} \rightarrow \infty,
$$


we have

$$
\lim _{z_{0} \rightarrow \infty} \widetilde{R}_{n-1}^{1}=\sum_{k=0}^{n-2}\left|\widetilde{P}_{k}\right|^{2} e^{-(n-1) \widetilde{Q}}
$$

where $\left\{\widetilde{P}_{k}\right\}$ are orthonormal polynomials with respect to the weight

$$
e^{-(n-1) \widetilde{Q}}=e^{-n Q} .
$$

Since the weight is the same for the polynomials $\left\{P_{k}\right\}$ and $\left\{\widetilde{P}_{k}\right\}$, we have

$$
\mathrm{Б}_{n}^{\langle\infty\rangle}=\sum_{k=0}^{n-1}\left|P_{k}\right|^{2} e^{-n Q}-\sum_{k=0}^{n-2}\left|P_{k}\right|^{2} e^{-n Q}=\left|P_{n-1}\right|^{2} e^{-n Q} .
$$

Combining this with Theorem 7.7.2, we conclude the proof.

\subsection{Further remarks on the cumulant method}

Here we continue our discussion of the cumulant method (see Section 1), and we compare our result with some other related work using this method.

In [30], Soshnikov studied linear statistics of the form trace $g_{n}-E\left(\operatorname{trace}_{n} g_{n}\right)$, where $g_{n}(t)=g\left(L_{n} t\right)$ and where $L_{n}$ is a fixed sequence with $L_{n} \rightarrow \infty, L_{n} / n \rightarrow 0$. The expectation here is understood with respect to the classical Weyl measure on $[-\pi, \pi)^{n}$, that is, we are considering the Gaussian unitary ensemble; $g: \mathbb{R} \rightarrow \mathbb{R}$ is a test function in the Schwarz class.

In [30], asymptotic normality is proved for these linear statistics using the cumulant method applied to the sine kernel, that is, the explicit correlation kernel in that case. The asymptotic variance of trace $g_{n}$ turns out to be finite and independent of the particular sequence $L_{n}$; it equals $1 / 2 \pi \int_{\mathbb{R}}|\hat{g}(t)|^{2}|t| \mathrm{d} t$, where $\hat{g}$ is the Fourier transform.

The method in [30], however, does not allow us to draw conclusions about the case $L_{n} \approx 1$; the assumption $L_{n} \rightarrow \infty$ is used in the proof of [30, Theorem 1, p. 1357], where limits of certain Riemann sums are identified.

We also want to mention the short proof of asymptotic normality due to Costin and Lebowitz [12]. In the situation of [12], one considers certain linear statistics which have infinite asymptotic variance. This infiniteness of the variance is then used to show decay of the cumulants of the corresponding normalized variables. (Thus the method in [12] necessarily breaks down in our situation, when the variance tends to a finite limit.)

The cumulant method has also been used in the theory of Gaussian analytic functions (see [26]). In this case, asymptotic normality was obtained for linear statistics whose variances converge to zero. In [28], the result was generalized to a setting of 
zeros of random holomorphic sections of high powers of a positive Hermitian line bundle over a Kähler manifold. (See [20] for further developments in the theory of Gaussian analytic functions.)

Acknowledgments. We are grateful to Alexei Borodin, Kurt Johansson, and Paul Wiegmann for help and useful discussions.

\section{References}

[1] Y. AMEUR, H. HEDENMALM, and N. MAKAROV, Berezin transform in polynomial Bergman spaces, Comm. Pure Appl. Math. 63, (2010), 1533-1584. MR 2742007

[2] - Random normal matrices and Ward's identities, in preparation.

[3] G. W. ANDERSON and O. ZEITOUNI, A CLT for a band matrix model, Probab. Theory Related Fields 134 (2006), 283 - 338. MR 2222385

[4] Z. D. BAI and J. W. SILVERSTEIN, CLT for linear spectral statistics of large-dimensional sample covariance matrices, Ann. Probab. 32 (2004), 533-605. MR 2040792

[5] R. J. BERMAN, Bergman kernels for weighted polynomials and weighted equilibrium measures of $\mathbb{C}^{n}$, Indiana Univ. Math. J. 58 (2009), 1921 - 1946. MR 2542983

[6] - Determinantal point processes and fermions on complex manifolds: Bulk universality, preprint, arXiv:08113341v1 [math.CV]

[7] R. J. BERMAN, B. BERNDTSSON, and J. SJÖSTRAND, A direct approach to Bergman kernel asymptotics for positive line bundles, Ark. Mat. 46 (2008), 197-217. MR 2430724

[8] P. BLEHER, B. SHIFFMAN, and S. ZELDITCH, Universality and scaling of correlations between zeros on complex manifolds, Invent. Math. 142 (2000), $351-395$. MR 1794066

[9] _ _ "Universality and scaling of zeros on symplectic manifolds" in Random Matrix Models and their Applications, Math. Sci. Res. Inst. Publ. 40, Cambridge Univ. Press, Cambridge, 2001, 31 - 69. MR 1842782

[10] CH. A. CHARALAMBIDES, Enumerative Combinatorics, CRC Press Series on Discrete Mathematics and its Applications, Chapman \& Hall/CRC, Boca Raton, Florida, 2002. MR 1937238

[11] L.-L. CHAU and O. ZABORONSKY, On the structure of correlation functions in the normal matrix model, Comm. Math. Phys. 196 (1998), 203-247. MR 1643533

[12] O. COSTIN and J. LEBOWITZ, Gaussian fluctuations in random matrices. Phys. Rev. Lett. 75 (1995), 69-72.

[13] P. DIACONIS and S. N. EVANS, Linear functionals of eigenvalues of random matrices, Trans. Amer. Math. Soc. 353 (2001), 2615 - 2633. MR 1828463

[14] P. ELBAU and G. FELDER, Density of eigenvalues of random normal matrices, Comm. Math. Phys. 259 (2005), 433 - 450. MR 2172690 
[15] P. ETINGOF and X. MA, Density of eigenvalues of random normal matrices with an arbitrary potential, and of generalized normal matrices, SIGMA Symmetry Integrability Geom. Methods Appl. 3 (2007), article 048. MR 2299849

[16] P. J. FORRESTER, Fluctuation formula for complex random matrices, J. Phys. A 32 (1999), 159-163. MR 1687948

[17] A. GUIONNET, Large deviations, upper bounds and central limit theorems for non-commutative functionals of Gaussian large random matrices, Ann. Inst. H. Poincaré Probab. Stat. 38 (2002), 341 - 384. MR 1899457

[18] H. HEDENMALM and N. MAKAROV, Quantum Hele-Shaw flow, preprint, arXiv:math/0411437v1 [math.PR]

[19] H. HEDENMALM and S. SHIMORIN, Hele-Shaw flow on hyperbolic surfaces, J. Math. Pures Appl. (9) 81 (2002), 187-222. MR 1894061

[20] J. B. HOUGH, M. KRISHNAPUR, Y. PERES, and B. VIRÁG, Zeros of Gaussian analytic functions and determinantal point processes, Univ. Lecture Ser. 51, Amer. Math. Soc., Providence, 2009. MR 2552864

[21] K. JOHANSSON, On random matrices from the classical compact groups, Ann. of Math. (2) 145 (1997), 519-545. MR 1454702

[22] - On fluctuations of eigenvalues of random Hermitian matrices, Duke Math. J. 91 (1998), 151 - 204. MR 1487983

[23] M. L. MEHTA, Random Matrices, 2nd ed., Academic Press, Boston, 1991. MR 1083764

[24] B. RIDER and B. VIRÁG, Complex determinantal processes and $H^{1}$ noise, Electron. J. Probab. 12 (2007), 1238 - 1257. MR 2346510

[25] - The noise in the circular law and the Gaussian free field, Int. Math. Res. Not. IMRN 2007, no. 2, art. ID rnm006. MR 2361453

[26] E. B. SAFF and V. TOTIK, Logarithmic Potentials with External Fields, Grundlehren Math. Wiss. 316, Springer, Berlin, 1997. MR 1485778

[27] M. SAKAI, Regularity of a boundary having a Schwarz function, Acta Math. 166 (1991), 263-297. MR 1097025

[28] B. SHIFFMAN and S. ZELDITCH, Number variance of random zeros on complex manifolds, II: Smooth statistics, Pure Appl. Math. Q. 6 (2010), 1145-1167. MR 2742043

[29] M. SODIN and B. TSIRELSON, Random complex zeroes, I, Asymptotic normality, Israel J. Math. 144 (2004), 125 - 149. MR 2121537

[30] A. SOSHNIKOV, The central limit theorem for local linear statistics in classical compact groups and related combinatorial identities, Ann. Probab. 28 (2000), 1353 - 1370. MR 1797877

[31] - Gaussian limits for determinantal random point fields. Ann. Probab. 30 (2002), 171-187. MR 1894104

[32] K. WIEAND, Eigenvalue distributions of random unitary matrices, Probab. Theory Related Fields 123, (2002), 202-224. MR 1900322

[33] A. ZABRODIN, "Matrix models and growth processes: From viscous flows to the quantum Hall effect" in Applications of Random Matrices in Physics, NATO Sci. Ser. II Math. Phys. Chem. 221, Springer, Dordrecht, 2006, 261 -318. MR 2232116 


\author{
Ameur \\ Department of Mathematics, Luleå University of Technology, 97187 Luleå, \\ Sweden; yacin.ameur@gmail.com \\ Hedenmalm \\ Department of Mathematics, Royal Institute of Technology, S-100 44 Stockholm, Sweden; \\ haakanh@math.kth.se

\section{Makarov} \\ Department of Mathematics, California Institute of Technology, Pasadena, California 91125, \\ USA; makarov@caltech.edu
}


